\title{
Pros and cons of using a standard protocol to test germination of alpine species
}

\author{
Vera Margreiter (D) Konrad Pagitz · Christian Berg • Patrick Schwager • \\ Brigitta Erschbamer
}

Received: 12 February 2020/Accepted: 28 July 2020/Published online: 3 August 2020

(C) The Author(s) 2020

\begin{abstract}
Storing seeds in seed banks is an effective way to preserve plant diversity and conserve species. An essential step towards a valuable conservation is the validation of germination. This study presents a germination screening of seeds from 255 species of the European Eastern Alps, which were to be stored at the Millennium Seed Bank (Kew, UK). The final germination percentage (FGP) was determined using a standard protocol in the laboratory. Species were classified according to species rarity, plant
\end{abstract}

Communicated by Thomas Abeli.

Electronic supplementary material The online version of this article (https://doi.org/10.1007/s11258-020-01061-w) contains supplementary material, which is available to authorized users.

V. Margreiter $(\bowtie) \cdot$ K. Pagitz · B. Erschbamer Department of Botany, University of Innsbruck, Sternwartestr. 15, 6020 Innsbruck, Austria e-mail: vera.margreiter4@gmail.com

K. Pagitz

e-mail: konrad.pagitz@uibk.ac.at

B. Erschbamer

e-mail: brigitta.erschbamer@uibk.ac.at

C. Berg · P. Schwager

Institute of Biology, Department of Plant Science, University of Graz, Holteigasse 6, $6020 \mathrm{Graz}$, Austria e-mail: christian.berg@uni-graz.at

P. Schwager

e-mail: p.schwager@gmx.at community, occurrence at elevation belts, bedrock types, as well as CSR strategies, and further, seed mass was examined. We could not find statistically significant differences of FGP within these classes, but $74.9 \%$ of all tested species germinated using the standard protocol, and half of them had FGP $\geq 20.1-100 \%$. A treatment with gibberellic acid enhanced the germination in half of the species to which this treatment was applied. Common families in alpine regions, i.e. Asteraceae, Poaceae and Saxifragaceae were highlighted in terms of their germination behaviour. The results provide an evaluation of the application of standard protocols to a broad Alpine species pool on the one hand, and on the other hand, provide ecological insights of the species tested. Germination is not only one of the most important events of the reproductive cycle of plants but could also be a key feature in species' responses to changing environmental conditions.

Keywords Seed bank $\cdot$ Eastern alps $\cdot$ Plant community $\cdot$ Rarity $\cdot$ Seed mass $\cdot$ Strategy

\section{Introduction}

Seed germination is one of the essential processes in a plants' life cycle (Harper 1977; Fenner and Thompson 2005). It is highly influenced by individual species' requirements (Baskin and Baskin 2014), 
characteristics of species' niches (Schwienbacher et al. 2012; Rosbakh and Poschlod 2015; JiménezAlfaro et al. 2016; Tudela-Isanta et al. 2018a, b), and the environmental conditions during seed development (Donohue et al. 2010; Bernareggi et al. 2016; Fernández-Pascual et al. 2017). With increasing temperatures in the Alps (Gobiet et al. 2014), knowledge on germination traits have become highly important. Generally, for common species, germination may be enhanced by increased temperatures (Milbau et al. 2009; Mondoni et al. 2012; Rosbakh and Poschlod 2015; Orsenigo et al. 2015). However, specific germination requirements (Graae et al. 2008; Shevtsova et al. 2009) and local adaptation (Kawecki and Ebert 2004; Mondoni et al. 2009) are bottlenecks for the growth of plant populations in a changing environment.

Alpine plant species require relatively high temperatures for germination (Bliss 1958; Amen 1966; Billings and Mooney 1968; Cavieres and Arroyo 2000; Giménez-Benavides et al. 2005; Graae et al. 2008; Schwienbacher et al. 2011; Mondoni et al. 2012; Baskin and Baskin 2014), whereas species below treeline use a broader temperature range (Walder and Erschbamer 2015; Fernández-Pascual et al. 2017). If so, increasing temperatures in the course of climate warming could be beneficial for both, alpine species and upward-migrating lowland species, in contrast to habitat specialists (Casazza et al. 2014) and species with specific dormancy strategies (Mondoni et al. 2012, 2018). Field studies on that matter were hardly performed and lab studies lack a comprehensive screening of species from different elevations and plant communities in the Alps (Mondoni et al. 2011, 2012; Isselin-Nondedeu and Bédécarrats 2013; Orsenigo et al. 2015; Walder and Erschbamer 2015; Tudela-Isanta et al. 2018a, b). Here, a broad germination project can close this gap.

The germination temperature can be regarded as a proxy for the germination niche of a species ('regeneration niche' according to Grubb 1977), which represents the ability of the species to adapt to environmental conditions (Rosbakh and Poschlod 2015; Jiménez-Alfaro et al. 2016). To investigate optimal seed germination temperatures, several temperature regimes (Mondoni et al. 2012) and seed pretreatments (Fernández-Pascual et al. 2017) are needed. In cases where seeds, time or working space is limited, a standard protocol with only one temperature regime can be used. This procedure is practiced, for example, when testing the germination ability of seed lots that are to be stored in ex situ seed banks. It remains questionable whether such standard protocols can provide reasonable data on the germination ability of species and whether the results allow an ecological interpretation.

One factor that hinders the success of germination tests is seed dormancy (Baskin and Baskin 2004, 2014; Finch-Savage and Leubner-Metzger 2006). It was found that physiological dormancy occurs frequently in alpine species (Schwienbacher et al. 2011). Here, a distinction must be made between non-deep, intermediate and deep physiological dormancy (Baskin and Baskin 2004). Non-deep physiological dormancy can be broken by dry-cold storage (Wang et al. 2010). This is done by storing the seeds in paper bags in a refrigerator, which is a very simple method of releasing dormancy. In contrast, breaking dormancy in seeds with medium or low physiological dormancy requires more effort, e.g. via stratification. In this method, the seeds are stored under wet-cold conditions for a certain period of time prior to a germination test (Baskin and Baskin 2004, 2014). Furthermore, physical, or morphological dormancy and all combinations can occur. Physical dormancy is a typical feature of hard-coated seeds, such as of Fabaceae. To release this type of dormancy, the seeds can be scarified so that water can penetrate through the damaged seed coat. Morphological dormancy occurs in seeds with immature embryos. These seeds require a post-ripening phase and often some additional treatments, such as a series of cold and warm treatments or vice versa, to break the dormancy (Baskin and Baskin 2014). A generally simple and timesaving method is treatment with gibberellic acid $\left(\mathrm{GA}_{3}\right)$. This method is recommended by ENSCONET (2009a; b) when a standard protocol is used or when the type of dormancy is unknown.

Among seed traits, seed mass seems to influence germination (Schwienbacher and Erschbamer 2001; $\mathrm{Bu}$ et al. 2007; Erschbamer et al. 2010; Liu et al. 2013), whereby large seeds are considered to have a superior germination and survival. However, contradicting results are found in the literature, noting only weak or no influence of seed mass on germination (Vera 1997; Eriksson 1999; Schwienbacher et al. 2012; Walder and Erschbamer 2015). 
The CSR strategy concept of Grime (1979) classifies the life strategies of plants as stress tolerant (S), competitive (C), ruderal (R) and intermediate strategists (e.g. CSR). Ruderal strategists do not have specific requirements for germination, while stress tolerant species may be less flexible and more demanding. In alpine environments, stress tolerant species represent the most common strategy group (Caccianiga et al. 2006).

This study presents the results of a broad-based germination screening of 255 Alpine plant species from the European Eastern Alps, including species from lowlands (dry grasslands, montane meadows), subalpine dwarf-shrub heaths, forest understorey species, species of wet habitats, tall herbs, species of alpine grasslands, rock crevices and scree sites. Seeds were collected by two partners of the "Alpine Seed Conservation and Research Network" (https://www. alpineseedconservation.eu/). The network was founded as a partner of the Millennium Seed Bank Partnership of the Royal Botanic Gardens, Kew (UK). One goal of this institution is to "store seeds of $25 \%$ of the world's flora by 2020" (Müller et al. 2017, https:// www.kew.org/science/). Contemporaneously, the study of germination behaviour is one of the main tasks of current seed ecology research, with the aim to evaluate the needs of species, local adaptation and the suitability of a species for ex situ conservation (Müller et al. 2017).

Having access to various species from different habitats and elevations, the study aimed to test the germination success of these different species under a standard protocol. Only one day/night temperature regime was used to evaluate if such an approach delivers appropriate germination data for an ex situ conservation. We were interested in finding out whether the results of our standard protocol provide evidence for adaptive mechanisms of species distribution along elevation gradients and habitats, and whether conclusions can be drawn about functional effects that influence the final germination percentage (FGP). Based on this focus, we addressed the following questions: (a) Does germination differ between common, scattered, rare and endemic species? (b) Does the FGP differ between species from different plant communities and (c) from different elevation belts (e.g. alpine, subalpine and montane/colline)? (d) Are there differences between species from siliceous and calcareous bedrock? (e) Is it possible to assign a germination pattern to the CSR strategy types sensu Grime (1979)? (f) Is there a correlation between FGP and seed mass? (g) Does treatment with gibberellic acid $\left(\mathrm{GA}_{3}\right)$ enhance FGP? (h) Do important families have a consistent germination pattern among their genera/species? Answers of these questions improve the interpretation of germination data from standard protocols in terms of ecological significance and can also be useful when predicting species responses to global warming.

\section{Material and methods}

\section{Seed collection}

Seeds were collected in the European Eastern Alps with two centres in Austria, one in the western part (Tyrol) and one in the south-eastern part (Styria). The test species (Table 1) were selected in agreement with the Millennium Seed Bank (MSB), representing 255 characteristic Alpine taxa from different plant communities, endemic/rare species, and species that were not already stored in the MSB. Nomenclature of the species and affiliation to families follow Fischer et al. (2008). The seed collection was carried out in the summers of 2016, 2017 and 2018. In all three years, an above-average number of warm days combined with above-average periods of sunshine were recorded in Austria by the research institute ZAMG (https://www. zamg.ac.at). The summer of 2016 was a warm and wet summer, the summer of 2017 was wet in mountain areas, while the summer of 2018 was recorded as a summer with above-average dry periods (ZAMG 2016, 2017, 2018). Collections were done in elevations from $600 \mathrm{~m}$ above sea level (a.s.1.) to $2700 \mathrm{~m}$ a.s.l. with a central focus at the subalpine and alpine elevation belt (1900-2400 m a.s.1.). According to international standards (ENSCONET 2009a), collections were performed at the time of natural dispersal. The major fraction of the cleaned seeds was sent to the MSB. A small fraction of each collection was stored dry in paper bags in a refrigerator at $+4{ }^{\circ} \mathrm{C}$ (average relative humidity of a refrigerator $\sim 40 \%$ ) until the germination tests started. The storage period lasted between 1 and 7 months. Fruits such as achenes, legumes or caryopses were treated as seeds. 
Table 1 Test species in alphabetical order, their family affiliation and rarity classification following Fischer et al. (2008)

\begin{tabular}{|c|c|c|c|c|c|c|c|c|c|c|c|}
\hline Species & Family & $\begin{array}{l}\text { Rar- } \\
\text { ity }\end{array}$ & $\begin{array}{l}\text { Commun- } \\
\text { ity }\end{array}$ & $\mathrm{T}$ & $\mathrm{R}$ & CSR & $\begin{array}{l}\text { Seed } \\
\text { mass }\end{array}$ & $\begin{array}{l}\text { FGP } \\
(\%)\end{array}$ & $\begin{array}{l}\text { SE } \\
\text { FGP }\end{array}$ & $\begin{array}{l}\text { GA } \\
\text { FGP }(\%)\end{array}$ & $\begin{array}{l}\text { SE } \\
\text { GA }\end{array}$ \\
\hline Achillea atrata & Asteraceae & Sca & ROSC & 1 & 5 & $\operatorname{css}$ & 0.242 & 92.80 & 3.44 & & \\
\hline Achillea clavennae* & Asteraceae & Sca & GRASS & 1.5 & 5 & $\operatorname{css}$ & 0.447 & 70.40 & 3.71 & & \\
\hline Achillea clusiana* & Asteraceae & end & ROSC & 1.5 & 5 & $\operatorname{css}$ & 0.326 & 81.60 & 6.76 & & \\
\hline Achillea moschata & Asteraceae & Com & ROSC & 1 & 2 & $\operatorname{css}$ & 0.220 & 51.72 & 5.59 & & \\
\hline Aconitum napellus & Ranunculaceae & Com & TALL & 3 & 3 & $\operatorname{ccs}$ & 3.100 & 0.00 & 0.00 & 1.80 & 1.11 \\
\hline Aconitum variegatum & Ranunculaceae & Com & TALL & 3 & 5 & $\operatorname{ccs}$ & 0.843 & 0.00 & 0.00 & 0.00 & 0.00 \\
\hline Adenostyles alliariae* & Asteraceae & Com & TALL & 2 & 3 & $\operatorname{ccs}$ & 1.258 & 45.60 & 5.60 & & \\
\hline Agrostis alpina & Poaceae & Sca & GRASS & 1.5 & 4 & crs & 0.277 & 26.40 & 4.12 & & \\
\hline Agrostis rupestris* & Poaceae & Sca & GRASS & 1 & 2 & crs & 0.108 & 60.80 & 1.50 & & \\
\hline Allium schoenoprasum* & Alliaceae & Sca & GRASS & 2.5 & 4 & crs & 0.990 & 56.80 & 6.97 & & \\
\hline Androsace lactea & Primulaceae & Sca & GRASS & 2.5 & 5 & crs & 1.013 & 0.00 & 0.00 & 15.58 & 4.66 \\
\hline Androsace obtusifolia & Primulaceae & Com & GRASS & 1.5 & 2 & $\operatorname{css}$ & 0.730 & 0.00 & 0.00 & 0.80 & 0.80 \\
\hline Antennaria carpatica & Asteraceae & Sca & GRASS & 1.5 & 3 & crs & 0.080 & 20.80 & 5.85 & & \\
\hline Antennaria dioica & Asteraceae & Sca & SHFO & 2 & 2 & crs & 0.047 & 86.40 & 3.25 & & \\
\hline Anthericum ramosum & Anthericaceae & Sca & DRYM & 3.5 & 4 & crs & 4.790 & 0.00 & 0.00 & 0.00 & 0.00 \\
\hline $\begin{array}{l}\text { Anthyllis vulneraria ssp. } \\
\quad \text { alpicola }\end{array}$ & Fabaceae & Com & GRASS & 1.5 & 5 & crs & 2.366 & 12.80 & 1.50 & 0.00 & 0.00 \\
\hline Aposeris foetida & Asteraceae & Sca & SHFO & 2.5 & 4 & $\operatorname{ccs}$ & 2.170 & 1.60 & 1.55 & 64.24 & 3.28 \\
\hline Arabidopsis halleri & Brassicaceae & Sca & MEAD & 2.5 & 2 & crs & 0.160 & 77.60 & 7.96 & & \\
\hline Arabis ciliata* & Brassicaceae & Sca & ROSC & 2.5 & 4 & crs & 0.111 & 92.80 & 5.43 & & \\
\hline Arabis stellulata & Brassicaceae & Sca & ROSC & 1.5 & 5 & rss & 0.234 & 4.74 & 2.31 & 58.80 & 4.96 \\
\hline Arenaria marschlinsii* & Brassicaceae & Rar & WET & 1 & 3 & rss & n.a & 95.20 & 1.50 & & \\
\hline Armeria alpina* & Plumbaginaceae & Sca & ROSC & 1 & 2 & $\operatorname{css}$ & 2.206 & 18.40 & 3.71 & & \\
\hline Arnica montana & Asteraceae & Com & MEAD & 2 & 3 & crs & 1.670 & 82.40 & 5.00 & & \\
\hline Artemisia mutellina* & Asteraceae & Sca & ROSC & 1 & 3 & rss & 0.280 & 64.80 & 11.83 & & \\
\hline Aster alpinus & Asteraceae & Sca & GRASS & 2 & 3 & crs & 1.350 & 80.80 & 3.44 & & \\
\hline Astragalus australis & Fabaceae & Rar & GRASS & 1.5 & 4 & $\operatorname{ccs}$ & 3.110 & 15.20 & 2.33 & & \\
\hline Athamantha cretensis & Apiaceae & Sca & ROSC & 2 & 5 & $\operatorname{css}$ & 2.150 & 1.60 & 0.98 & & \\
\hline Atocion rupestre* & Caryophyllaceae & Com & ROSC & 2.5 & 2 & $\operatorname{css}$ & 0.074 & 43.20 & 6.50 & & \\
\hline Bartsia alpina & Orobanchaceae & Com & GRASS & 2 & 3 & rss & 0.425 & 0.00 & 0.00 & 59.38 & 3.25 \\
\hline Blysmus compressus & Poaceae & Sca & WET & 3 & 4 & crs & 0.905 & 10.40 & 3.49 & 0.00 & 0.00 \\
\hline Bothriochloa ischaemum & Poaceae & Sca & DRYM & 4.5 & 3 & crs & 0.600 & 42.40 & 0.98 & & \\
\hline Buphthalmum salicifolium* & Asteraceae & Com & DRYM & 3 & 4 & crs & 0.700 & 61.60 & 3.25 & & \\
\hline Calluna vulgaris & Ericaceae & Com & SHFO & 2.5 & 1 & $\operatorname{css}$ & 0.030 & 52.80 & 5.43 & & \\
\hline Campanula alpina & Campanulaceae & Rar & ROSC & 2 & 2 & $\operatorname{css}$ & 0.333 & 0.80 & 0.00 & 58.13 & 3.94 \\
\hline Campanula barbata* & Campanulaceae & Com & GRASS & 2 & 2 & crs & 0.050 & 49.60 & 4.66 & & \\
\hline Campanula cochleariifolia* & Campanulaceae & Com & ROSC & 2 & 5 & $\operatorname{css}$ & 0.045 & 7.00 & 2.41 & 34.73 & 7.62 \\
\hline Campanula pulla & Campanulaceae & end & GRASS & 1.5 & 4 & $\operatorname{css}$ & 0.132 & 1.60 & 0.98 & 0.00 & 0.00 \\
\hline Campanula rapunculus & Campanulaceae & Rar & DRYM & 4.5 & 3 & crs & 0.040 & 43.20 & 3.44 & & \\
\hline Campanula scheuchzeri & Campanulaceae & Com & GRASS & 1.5 & 3 & crs & 0.090 & 7.20 & 2.65 & 90.26 & 3.77 \\
\hline Carduus personata* & Asteraceae & Sca & TALL & 2.5 & 4 & $\mathrm{ccr}$ & 1.709 & 91.20 & 2.94 & & \\
\hline Carex brachystachys & Cyperaceae & Sca & GRASS & 2.5 & 5 & $\operatorname{css}$ & 0.496 & 0.00 & 0.00 & 0.00 & 0.00 \\
\hline
\end{tabular}


Table 1 continued

\begin{tabular}{|c|c|c|c|c|c|c|c|c|c|c|c|}
\hline Species & Family & $\begin{array}{l}\text { Rar- } \\
\text { ity }\end{array}$ & $\begin{array}{l}\text { Commun- } \\
\text { ity }\end{array}$ & $\mathrm{T}$ & $\mathrm{R}$ & CSR & $\begin{array}{l}\text { Seed } \\
\text { mass }\end{array}$ & $\begin{array}{l}\text { FGP } \\
(\%)\end{array}$ & $\begin{array}{l}\text { SE } \\
\text { FGP }\end{array}$ & $\begin{array}{l}\text { GA } \\
\text { FGP }(\%)\end{array}$ & $\begin{array}{l}\text { SE } \\
\text { GA }\end{array}$ \\
\hline Carex curvula & Cyperaceae & Com & GRASS & 1 & 2 & $\operatorname{ccs}$ & 2.120 & 0.00 & 0.00 & 0.00 & 0.00 \\
\hline Carex davalliana & Cyperaceae & Com & WET & 3 & 4 & $\operatorname{css}$ & 0.839 & 8.00 & 3.58 & 42.14 & 11.85 \\
\hline Carex echinata & Cyperaceae & Com & WET & 3 & 2 & css & 0.840 & 29.60 & 3.49 & & \\
\hline Carex ferruginea* & Cyperaceae & Com & GRASS & 2 & 4 & $\operatorname{ccs}$ & 1.210 & 28.00 & 2.83 & & \\
\hline Carex firma & Cyperaceae & Com & GRASS & 1.5 & 5 & $\operatorname{css}$ & 0.770 & 53.60 & 3.25 & & \\
\hline Carex frigida & Cyperaceae & Sca & WET & 1.5 & 4 & css & 0.320 & 2.50 & 2.50 & 5.00 & 2.24 \\
\hline Carex nigra & Cyperaceae & Com & WET & 2.5 & 2 & $\operatorname{css}$ & 0.760 & 0.00 & 0.00 & 0.00 & 0.00 \\
\hline Carex panicea & Cyperaceae & Com & WET & 3 & 4 & css & 2.473 & 0.00 & 0.00 & 0.00 & 0.00 \\
\hline Carex parviflora* & Cyperaceae & Sca & GRASS & 1 & 5 & crs & 0.450 & 7.20 & 1.96 & 79.68 & 4.94 \\
\hline Carex rostrata & Cyperaceae & Sca & WET & 3 & 2 & $\operatorname{css}$ & 1.500 & 17.60 & 6.01 & & \\
\hline Carex vesicaria & Cyperaceae & Sca & WET & 3.5 & 3 & $\operatorname{css}$ & 1.800 & 0.00 & 0.00 & 0.00 & 0.00 \\
\hline Carlina acaulis & Asteraceae & Com & DRYM & 3 & 2 & crs & 3.400 & 100.00 & 0.00 & & \\
\hline Centaurea pseudophrygia & Asteraceae & Sca & DRYM & 2.5 & 3 & $\operatorname{ccs}$ & n.a & 38.40 & 3.49 & & \\
\hline Centaurea stoebe & Asteraceae & Sca & DRYM & 4.5 & 4 & crs & 1.404 & 71.20 & 5.57 & & \\
\hline Centaurium erythraea & Gentianaceae & Sca & DRYM & 3.5 & 3 & rrs & 0.010 & 92.80 & 2.33 & & \\
\hline Cerastium carinthiacum & Caryophyllaceae & Sca & ROSC & 1.5 & 5 & $\operatorname{css}$ & 0.200 & 32.80 & 4.63 & & \\
\hline Cerastium eriophorum & Caryophyllaceae & Sca & GRASS & 1.5 & 4 & $\operatorname{css}$ & 0.288 & 54.40 & 8.82 & & \\
\hline Cervaria rivini & Apiaceae & Sca & TALL & 4 & 4 & $\operatorname{ccs}$ & 4.020 & 0.80 & 0.80 & 28.10 & 6.00 \\
\hline Chaerophyllum aureum & Apiaceae & Sca & TALL & 3 & 4 & $\mathrm{ccc}$ & 8.100 & 0.00 & 0.00 & 0.00 & 0.00 \\
\hline Chaerophyllum villarsii & Apiaceae & Sca & TALL & 2 & 3 & $\operatorname{ccs}$ & 7.666 & 0.00 & 0.00 & 0.00 & 0.00 \\
\hline Cirsium carniolicum & Asteraceae & Rar & TALL & 2.5 & 4 & $\operatorname{ccs}$ & 5.400 & 40.80 & 0.80 & & \\
\hline Cirsium erisithales & Asteraceae & Rar & TALL & 2.5 & 4 & $\operatorname{ccs}$ & 1.956 & 55.20 & 4.08 & & \\
\hline Cirsium spinosissimum & Asteraceae & Sca & TALL & 1.5 & 3 & $\operatorname{ccs}$ & 2.168 & 49.60 & 3.71 & & \\
\hline Comarum palustre & Rosaceae & Rar & WET & 3 & 2 & $\operatorname{ccs}$ & 0.320 & 1.60 & 1.60 & 6.50 & 1.57 \\
\hline Crepis alpestris & Asteraceae & Sca & MEAD & 2 & 5 & $\operatorname{ccs}$ & 2.319 & 85.60 & 3.71 & & \\
\hline Crepis aurea & Asteraceae & Com & MEAD & 2 & 3 & $\operatorname{ccs}$ & 0.856 & 26.40 & 8.73 & & \\
\hline Crepis conyzifolia* & Asteraceae & Rar & MEAD & 2 & 2 & $\operatorname{ccs}$ & 2.403 & 87.20 & 3.44 & & \\
\hline Crepis jacquinii & Asteraceae & Sca & ROSC & 1.5 & 5 & $\operatorname{css}$ & 0.897 & 92.00 & 2.83 & & \\
\hline Crepis kerneri & Asteraceae & Sca & GRASS & 1.5 & 5 & $\operatorname{css}$ & n.a & 86.40 & 0.98 & & \\
\hline Crepis paludosa & Asteraceae & Sca & WET & 3 & 3 & $\operatorname{ccs}$ & 0.652 & 59.20 & 4.27 & & \\
\hline Crepis pyrenaica* & Asteraceae & Sca & TALL & 2.5 & 4 & $\operatorname{ccs}$ & 3.097 & 36.80 & 6.37 & & \\
\hline Dactylorhiza incarnata & Orchidaceae & Sca & WET & 3.5 & 4 & crs & 0.001 & 0.00 & 0.00 & 0.00 & 0.00 \\
\hline Dianthus alpinus* & Caryophyllaceae & end & ROSC & 1 & 5 & $\operatorname{css}$ & 0.580 & 47.20 & 6.12 & & \\
\hline Dianthus superbus* & Caryophyllaceae & Sca & WET & 3.5 & 4 & crs & 0.583 & 68.00 & 5.06 & & \\
\hline Dianthus sylvestris & Caryophyllaceae & Sca & ROSC & 3 & 3 & $\mathrm{crs}$ & 0.850 & 48.00 & 4.38 & & \\
\hline Drosera rotundifolia & Droseraceae & Com & WET & 3 & 1 & sss & 0.020 & 0.00 & 0.00 & 12.00 & 3.00 \\
\hline Dryas octopetala & Rosaceae & Com & GRASS & 1.5 & 5 & crs & 0.372 & 83.20 & 4.96 & & \\
\hline Epilobium alpestre ${ }^{*}$ & Onagraceae & Com & TALL & 2.5 & 4 & $\operatorname{ccs}$ & 0.267 & 96.80 & 1.50 & & \\
\hline Epilobium anagallidifolium & Onagraceae & Sca & WET & 1.5 & 3 & $\operatorname{css}$ & 0.060 & 92.00 & 2.53 & & \\
\hline Epilobium hirsutum & Onagraceae & Sca & TALL & 3.5 & 4 & $\mathrm{ccc}$ & 0.100 & 99.20 & 0.80 & & \\
\hline Epilobium nutans $*$ & Onagraceae & Rar & WET & 2 & 2 & crs & 0.075 & 48.00 & 7.38 & & \\
\hline Epilobium palustre & Onagraceae & Sca & WET & 3 & 3 & crs & 0.090 & 4.80 & 2.94 & 72.72 & 5.01 \\
\hline
\end{tabular}


Table 1 continued

\begin{tabular}{|c|c|c|c|c|c|c|c|c|c|c|c|}
\hline Species & Family & $\begin{array}{l}\text { Rar- } \\
\text { ity }\end{array}$ & $\begin{array}{l}\text { Commun- } \\
\text { ity }\end{array}$ & $\mathrm{T}$ & $\mathrm{R}$ & CSR & $\begin{array}{l}\text { Seed } \\
\text { mass }\end{array}$ & $\begin{array}{l}\text { FGP } \\
(\%)\end{array}$ & $\begin{array}{l}\text { SE } \\
\text { FGP }\end{array}$ & $\begin{array}{l}\text { GA } \\
\text { FGP }(\%)\end{array}$ & $\begin{array}{l}\text { SE } \\
\text { GA }\end{array}$ \\
\hline Epipactis palustris* & Orchidaceae & Rar & WET & 3.5 & 4 & crs & 0.004 & 0.00 & 0.00 & 0.00 & 0.00 \\
\hline Erigeron atticus & Asteraceae & Rar & GRASS & 2 & 2 & crs & 0.234 & 65.60 & 7.65 & & \\
\hline Erigeron glabratus $*$ & Asteraceae & Com & GRASS & 1.5 & 5 & $\operatorname{css}$ & 0.199 & 58.40 & 5.31 & & \\
\hline Erigeron uniflorus* & Asteraceae & Sca & GRASS & 1 & 3 & $\operatorname{css}$ & 0.180 & 87.20 & 5.85 & & \\
\hline Eriophorum latifolium & Cyperaceae & com & WET & 3 & 4 & $\operatorname{css}$ & 0.654 & 0.80 & 0.80 & 1.67 & 1.67 \\
\hline Eriophorum vaginatum & Cyperaceae & rar & WET & 2.5 & 1 & $\operatorname{css}$ & 0.577 & 83.20 & 7.42 & & \\
\hline Erysimum rhaeticum & Brassicaceae & sca & GRASS & 3 & 2 & crs & n.a & 76.00 & 6.07 & & \\
\hline $\begin{array}{l}\text { Euphrasia officinalis } \\
\text { ssp. picta }\end{array}$ & Orobanchaceae & sca & GRASS & 2 & 3 & rrs & n.a & 0.80 & 0.80 & 11.52 & 3.91 \\
\hline Festuca picturata* & Poaceae & sca & GRASS & 1.5 & 2 & $\operatorname{ccs}$ & n.a & 47.20 & 6.50 & & \\
\hline Festuca pseudodura & Poaceae & com & GRASS & 1.5 & 2 & $\operatorname{ccs}$ & 0.334 & 29.60 & 9.26 & & \\
\hline Festuca varia & Poaceae & com & GRASS & 1.5 & 2 & $\operatorname{ccs}$ & 1.150 & 10.40 & 4.12 & 27.14 & 12.33 \\
\hline Galium anisophyllon & Rubiaceae & com & GRASS & 2 & 3 & $\operatorname{ccs}$ & 0.365 & 27.20 & 4.27 & & \\
\hline Gentiana acaulis & Gentianaceae & sca & GRASS & 1.5 & 2 & $\operatorname{css}$ & 0.452 & 0.00 & 0.00 & 38.70 & 2.59 \\
\hline Gentiana bavarica & Gentianaceae & sca & WET & 1.5 & 3 & $\operatorname{css}$ & 0.140 & 0.00 & 0.00 & 52.40 & 8.63 \\
\hline Gentiana clusii & Gentianaceae & sca & GRASS & 1.5 & 5 & $\operatorname{css}$ & 0.410 & 0.00 & 0.00 & 47.50 & 2.13 \\
\hline Gentiana lutea & Gentianaceae & rar & GRASS & 2.5 & 4 & $\operatorname{ccs}$ & 1.500 & 0.80 & 0.80 & 57.14 & 7.23 \\
\hline Gentiana nivalis & Gentianaceae & sca & GRASS & 1.5 & 3 & $\operatorname{css}$ & 0.081 & 0.00 & 0.00 & 21.56 & 4.64 \\
\hline Gentiana pannonica & Gentianaceae & sca & GRASS & 2 & 3 & $\operatorname{ccs}$ & 0.460 & 33.60 & 4.12 & & \\
\hline Gentiana pumila & Gentianaceae & sca & WET & 1.5 & 5 & $\operatorname{css}$ & 0.167 & 0.00 & 0.00 & 70.66 & 2.23 \\
\hline Gentiana utriculosa & Gentianaceae & sca & WET & 2.5 & 4 & crs & 0.060 & 0.00 & 0.00 & 77.60 & 6.01 \\
\hline Gentianella aspera* & Gentianaceae & com & GRASS & 2.5 & 4 & crs & 0.219 & 0.00 & 0.00 & 12.38 & 2.24 \\
\hline Globularia cordifolia* & Globulariaceae & com & GRASS & 2.5 & 5 & $\operatorname{css}$ & 0.530 & 32.80 & 3.44 & & \\
\hline Globularia nudicaulis & Globulariaceae & rar & GRASS & 2 & 4 & $\operatorname{ccs}$ & 0.720 & 15.20 & 3.88 & & \\
\hline Gnaphalium sylvaticum & Asteraceae & sca & SHFO & 3 & 2 & crs & 0.061 & 94.40 & 0.98 & & \\
\hline Gypsophila repens & Caryophyllaceae & sca & ROSC & 2 & 5 & crs & 0.710 & 88.86 & 2.35 & & \\
\hline Hedysarum hedysaroides & Fabaceae & sca & SHFO & 1.5 & 4 & $\operatorname{ccs}$ & 4.125 & 55.20 & 6.12 & & \\
\hline Helianthemum nummularium & Cistaceae & sca & GRASS & 4.5 & 4 & $\operatorname{ccs}$ & 1.100 & 1.60 & 1.60 & 2.40 & 0.98 \\
\hline Helictotrichon parlatorei & Poaceae & sca & GRASS & 1.5 & 4 & $\operatorname{css}$ & 1.789 & 0.80 & 0.80 & 1.60 & 0.98 \\
\hline Heracleum austriacum & Apiaceae & sca & SHFO & 2.5 & 4 & crs & 5.429 & 0.00 & 0.00 & 9.72 & 2.62 \\
\hline Hieracium hорреапит & Asteraceae & sca & SHFO & 2.5 & 2 & crs & 0.276 & 57.60 & 6.27 & & \\
\hline Hieracium intybaceum & Asteraceae & sca & ROSC & 2 & 2 & $\operatorname{css}$ & 0.647 & 7.20 & 2.33 & 68.80 & 3.88 \\
\hline Hieracium maculatum & Asteraceae & rar & DRYM & 3.5 & 3 & $\operatorname{css}$ & 0.523 & 47.20 & 7.09 & & \\
\hline Hieracium racemosum & Asteraceae & rar & SHFO & 4.5 & 2 & $\operatorname{css}$ & 0.511 & 56.80 & 6.62 & & \\
\hline Hieracium sphaerocephalum & Asteraceae & sca & GRASS & 1.5 & 2 & $\operatorname{css}$ & 0.128 & 29.60 & 3.49 & & \\
\hline Hieracium umbellatum & Asteraceae & sca & SHFO & 4 & 2 & $\operatorname{css}$ & 0.530 & 64.80 & 9.75 & & \\
\hline Hieracium villosum & Asteraceae & com & GRASS & 2 & 5 & $\operatorname{css}$ & 0.445 & 0.80 & 0.80 & 63.36 & 4.85 \\
\hline Homogyne alpina & Asteraceae & com & SHFO & 2 & 2 & crs & 0.946 & 30.40 & 7.86 & & \\
\hline Homogyne discolor* & Asteraceae & rar & GRASS & 1.5 & 4 & crs & 0.891 & 13.89 & 4.00 & 66.00 & 2.00 \\
\hline Hornungia alpina & Brassicaceae & com & ROSC & 1 & 4 & rss & 0.318 & 37.60 & 2.99 & & \\
\hline Hypochaeris uniflora* & Asteraceae & sca & MEAD & 2 & 2 & crs & 3.800 & 60.80 & 8.14 & & \\
\hline Juncus trifidus & Juncaceae & sca & GRASS & 1.5 & 1 & $\operatorname{css}$ & 0.176 & 0.00 & 0.00 & 14.56 & 2.23 \\
\hline
\end{tabular}


Table 1 continued

\begin{tabular}{|c|c|c|c|c|c|c|c|c|c|c|c|}
\hline Species & Family & $\begin{array}{l}\text { Rar- } \\
\text { ity }\end{array}$ & $\begin{array}{l}\text { Commun- } \\
\text { ity }\end{array}$ & $\mathrm{T}$ & $\mathrm{R}$ & CSR & $\begin{array}{l}\text { Seed } \\
\text { mass }\end{array}$ & $\begin{array}{l}\text { FGP } \\
(\%)\end{array}$ & $\begin{array}{l}\text { SE } \\
\text { FGP }\end{array}$ & $\begin{array}{l}\text { GA } \\
\text { FGP }(\%)\end{array}$ & $\begin{array}{l}\text { SE } \\
\text { GA }\end{array}$ \\
\hline Knautia maxima & Dipsacaceae & com & TALL & 3 & 3 & $\operatorname{ccs}$ & 3.225 & 12.00 & 3.58 & 96.36 & 2.23 \\
\hline Kobresia myosuroides & Cyperaceae & sca & GRASS & 1.5 & 3 & $\operatorname{ccs}$ & 0.655 & 0.80 & 0.80 & 19.68 & 4.01 \\
\hline Kobresia simpliciuscula* & Cyperaceae & rar & WET & 1.5 & 4 & $\operatorname{css}$ & 0.491 & 88.00 & 3.10 & & \\
\hline Koeleria hirsuta & Poaceae & rar & ROSC & 1.5 & 2 & $\operatorname{css}$ & n.a & 85.33 & 6.67 & & \\
\hline Laserpitium halleri & Apiaceae & sca & GRASS & 2 & 2 & $\operatorname{ccs}$ & 6.829 & 0.00 & 0.00 & 1.00 & 1.00 \\
\hline Laserpitium krapfii* & Apiaceae & rar & SHFO & 3 & 3 & $\operatorname{ccs}$ & n.a & 0.00 & 0.00 & 0.00 & 0.00 \\
\hline Laserpitium latifolium & Apiaceae & sca & SHFO & 3 & 4 & $\operatorname{ccs}$ & 9.680 & 0.00 & 0.00 & 0.00 & 0.00 \\
\hline Leontopodium alpinum & Asteraceae & rar & GRASS & 1.5 & 4 & $\operatorname{ccs}$ & 0.127 & 74.40 & 3.25 & & \\
\hline Leucanthemopsis alpina & Asteraceae & com & GRASS & 1 & 2 & $\operatorname{ccs}$ & 0.380 & 29.29 & 3.45 & & \\
\hline Leucanthemum atratum & Asteraceae & end & GRASS & 1.5 & 5 & $\operatorname{css}$ & 0.578 & 95.20 & 1.50 & & \\
\hline Leucanthemum vulgare & Asteraceae & com & MEAD & 3 & 3 & crs & 0.420 & 92.80 & 1.96 & & \\
\hline Linaria alpina & Antirrhinaceae & com & ROSC & 1.5 & 4 & rrs & 0.278 & 0.00 & 0.00 & 89.38 & 1.69 \\
\hline Linum alpinum & Linaceae & sca & GRASS & 2 & 4 & crs & 1.883 & 52.00 & 3.10 & & \\
\hline Luzula alpina & Juncaceae & sca & GRASS & 1.5 & 2 & crs & 0.257 & 86.40 & 2.71 & & \\
\hline Luzula alpinopilosa* & Juncaceae & sca & GRASS & 1 & 2 & $\operatorname{css}$ & 0.200 & 54.40 & 2.99 & & \\
\hline Luzula glabrata* & Juncaceae & sca & GRASS & 1.5 & 4 & $\operatorname{css}$ & 0.281 & 6.40 & 3.49 & & \\
\hline Luzula spicata & Juncaceae & sca & GRASS & 1.5 & 2 & crs & 0.340 & 0.00 & 0.00 & 0.80 & 0.80 \\
\hline Luzula sudetica* & Juncaceae & rar & WET & 2 & 2 & crs & 0.373 & 67.20 & 4.63 & & \\
\hline Menyanthes trifoliata & Menyanthaceae & rar & WET & 3 & 3 & $\operatorname{css}$ & 2.400 & 0.00 & 0.00 & 15.20 & 2.65 \\
\hline Minuartia austriaca* & Caryophyllaceae & sca & ROSC & 1.5 & 5 & $\operatorname{css}$ & 0.290 & 0.80 & 0.80 & 0.76 & 0.80 \\
\hline Minuartia cherlerioides & Caryophyllaceae & rar & ROSC & 1 & 5 & $\operatorname{css}$ & 0.061 & 10.40 & 6.76 & 82.24 & 5.66 \\
\hline Minuartia sedoides & Caryophyllaceae & sca & GRASS & 1 & 3 & $\operatorname{css}$ & 0.258 & 7.20 & 2.65 & & \\
\hline Moehringia ciliata & Caryophyllaceae & sca & ROSC & 1 & 4 & $\operatorname{css}$ & 0.387 & 0.00 & 0.00 & 1.60 & 0.98 \\
\hline Molinia arundinacea & Poaceae & sca & SHFO & 3.5 & 4 & $\operatorname{css}$ & n.a & 4.00 & 1.79 & 12.75 & 2.66 \\
\hline Mutellina adonidifolia* & Apiaceae & com & SHFO & 1.5 & 2 & $\operatorname{ccs}$ & 2.185 & 0.00 & 0.00 & 0.00 & 0.00 \\
\hline Noccaea crantzii & Brassicaceae & end & GRASS & 2 & 5 & crs & 0.828 & 7.20 & 1.50 & 90.30 & 3.73 \\
\hline Oreochloa disticha* & Poaceae & sca & GRASS & 1 & 1 & crs & 0.373 & 20.00 & 4.90 & & \\
\hline Orobanche flava & Orobanchaceae & sca & TALL & 2.5 & 4 & rss & 0.006 & 0.00 & 0.00 & 0.00 & 0.00 \\
\hline Orobanche salviae & Orobanchaceae & rar & SHFO & 3 & 4 & rss & n.a & 0.00 & 0.00 & 0.00 & 0.00 \\
\hline Oxytropis montana* & Fabaceae & sca & GRASS & 1.5 & 5 & $\operatorname{ccs}$ & 3.253 & 6.40 & 0.98 & 0.00 & 0.00 \\
\hline Pachypleurum mutellinoides & Apiaceae & rar & GRASS & 1 & 3 & crs & 1.020 & 0.80 & 0.80 & 7.26 & 2.19 \\
\hline Papaver alpinum* & Papaveraceae & sca & ROSC & 1.5 & 4 & sss & 0.143 & 15.20 & 3.44 & 84.15 & 1.64 \\
\hline Parnassia palustris* & Parnassiaceae & sca & WET & 2 & 4 & $\operatorname{css}$ & 0.030 & 60.00 & 6.69 & & \\
\hline Pedicularis palustris & Orobanchaceae & rar & WET & 3 & 3 & $\operatorname{css}$ & 0.829 & 0.00 & 0.00 & 8.00 & 2.19 \\
\hline Pedicularis portenschlagii & Orobanchaceae & end & GRASS & 1 & 3 & $\operatorname{css}$ & 0.646 & 1.60 & 0.98 & 81.28 & 3.76 \\
\hline Pedicularis recutita & Orobanchaceae & sca & TALL & 2 & 4 & $\operatorname{css}$ & 0.820 & 0.00 & 0.00 & 57.76 & 4.22 \\
\hline Pedicularis rostratocapitata & Orobanchaceae & com & GRASS & 1.5 & 5 & $\operatorname{css}$ & 1.055 & 0.00 & 0.00 & 53.50 & 3.32 \\
\hline Petasites paradoxus & Asteraceae & com & TALL & 2 & 5 & $\operatorname{ccs}$ & 0.476 & 2.40 & 1.60 & & \\
\hline Peucedanum oreoselinum* & Apiaceae & sca & SHFO & 4.5 & 3 & $\operatorname{ccs}$ & 4.520 & 56.80 & 6.37 & & \\
\hline Peucedanum ostruthium & Apiaceae & sca & TALL & 2 & 3 & $\mathrm{ccc}$ & 1.208 & 26.40 & 4.12 & & \\
\hline Peucedanum verticillare & Apiaceae & rar & TALL & 4 & 4 & $\operatorname{ccs}$ & n.a & 72.00 & 5.51 & & \\
\hline Phleum hirsutum & Poaceae & sca & TALL & 2 & 4 & $\operatorname{ccs}$ & 0.169 & 89.60 & 2.99 & & \\
\hline
\end{tabular}


Table 1 continued

\begin{tabular}{|c|c|c|c|c|c|c|c|c|c|c|c|}
\hline Species & Family & $\begin{array}{l}\text { Rar- } \\
\text { ity }\end{array}$ & $\begin{array}{l}\text { Commun- } \\
\text { ity }\end{array}$ & $\mathrm{T}$ & $\mathrm{R}$ & CSR & $\begin{array}{l}\text { Seed } \\
\text { mass }\end{array}$ & $\begin{array}{l}\text { FGP } \\
(\%)\end{array}$ & $\begin{array}{l}\text { SE } \\
\text { FGP }\end{array}$ & $\begin{array}{l}\text { GA } \\
\text { FGP }(\%)\end{array}$ & $\begin{array}{l}\text { SE } \\
\text { GA }\end{array}$ \\
\hline Phleum rhaeticum & Poaceae & com & TALL & 2 & 3 & $\operatorname{ccs}$ & 0.464 & 67.13 & 5.34 & & \\
\hline Phyteuma betonicifolium & Campanulaceae & sca & TALL & 2 & 2 & $\operatorname{ccs}$ & 0.045 & 3.20 & 1.50 & 67.82 & 14.11 \\
\hline Phyteuma confusum & Campanulaceae & sca & GRASS & 1.5 & 2 & $\operatorname{css}$ & 0.043 & 8.00 & 2.53 & 89.78 & 4.10 \\
\hline Phyteuma globulariifolium* & Campanulaceae & sca & GRASS & 1 & 2 & $\operatorname{css}$ & 0.052 & 3.20 & 1.50 & 81.23 & 4.61 \\
\hline Phyteuma hemisphaericum & Campanulaceae & com & GRASS & 1.5 & 1 & $\operatorname{css}$ & 0.101 & 21.60 & 4.49 & & \\
\hline Phyteuma orbiculare* & Campanulaceae & sca & MEAD & 2.5 & 4 & $\operatorname{css}$ & 0.215 & 0.00 & 0.00 & 74.97 & 1.64 \\
\hline Phyteuma ovatum & Campanulaceae & sca & TALL & 2 & 3 & $\operatorname{ccs}$ & 0.180 & 0.00 & 0.00 & 84.68 & 4.56 \\
\hline Pinguicula leptoceras* & Lentibulariaceae & $\operatorname{rar}$ & WET & 1.5 & 3 & sss & 0.020 & 4.00 & 2.19 & 66.15 & 5.79 \\
\hline Plantago alpina & Plantaginaceae & rar & GRASS & 1.5 & 2 & $\operatorname{css}$ & 0.600 & 42.40 & 3.71 & & \\
\hline Poa alpina & Poaceae & com & GRASS & 1.5 & 3 & crs & 0.354 & 48.80 & 7.74 & & \\
\hline Poa chaixii & Poaceae & rar & TALL & 2.5 & 2 & $\operatorname{ccs}$ & 0.503 & 5.60 & 2.71 & 95.00 & 2.74 \\
\hline Poa minor & Poaceae & sca & GRASS & 1 & 5 & $\operatorname{css}$ & 0.299 & 84.80 & 6.37 & & \\
\hline Potentilla aurea* & Rosaceae & com & MEAD & 1.5 & 2 & $\operatorname{ccs}$ & 0.368 & 35.20 & 3.88 & & \\
\hline Potentilla brauneana & Rosaceae & rar & GRASS & 1 & 5 & $\operatorname{css}$ & 0.615 & 0.80 & 0.80 & 46.96 & 4.43 \\
\hline Potentilla clusiana* & Rosaceae & end & ROSC & 1.5 & 5 & $\operatorname{css}$ & 0.373 & 25.60 & 5.74 & & \\
\hline Potentilla frigida* & Rosaceae & rar & GRASS & 1 & 2 & $\operatorname{css}$ & 0.200 & 3.20 & 0.80 & 6.93 & 2.26 \\
\hline Potentilla grandiflora* & Rosaceae & sca & GRASS & 1.5 & 2 & $\operatorname{ccs}$ & 0.449 & 8.80 & 3.20 & 48.00 & 13.80 \\
\hline Primula auricula & Primulaceae & sca & GRASS & 1.5 & 5 & $\operatorname{css}$ & 0.250 & 0.00 & 0.00 & 87.14 & 3.18 \\
\hline Primula clusiana* & Primulaceae & end & GRASS & 1.5 & 5 & $\operatorname{css}$ & 0.315 & 32.00 & 8.10 & & \\
\hline Primula elatior & Primulaceae & com & MEAD & 3 & 4 & $\operatorname{ccs}$ & 0.800 & 0.00 & 0.00 & 35.40 & 3.28 \\
\hline Primula farinosa & Primulaceae & rar & WET & 2 & 4 & $\operatorname{css}$ & 0.060 & 5.60 & 2.71 & 97.60 & 1.60 \\
\hline Primula glutinosa & Primulaceae & sca & GRASS & 1 & 2 & $\operatorname{css}$ & 0.121 & 5.60 & 1.60 & 16.00 & 8.00 \\
\hline Primula minima* & Primulaceae & com & GRASS & 1.5 & 2 & sss & 0.123 & 25.38 & 2.96 & & \\
\hline Pulsatilla alpina* & Ranunculaceae & sca & SHFO & 2 & 4 & crs & 6.680 & 0.00 & 0.00 & 69.10 & 8.29 \\
\hline $\begin{array}{l}\text { Pulsatilla alpina ssp. } \\
\text { Apiifolia* }\end{array}$ & Ranunculaceae & sca & SHFO & 2 & 2 & crs & n.a & 0.00 & 0.00 & 63.00 & 13.00 \\
\hline Ranunculus alpestris & Ranunculaceae & com & GRASS & 1.5 & 4 & $\operatorname{css}$ & 0.278 & 18.40 & 6.40 & & \\
\hline Ranunculus montanus & Ranunculaceae & com & MEAD & 2 & 4 & $\operatorname{ccs}$ & 1.547 & 0.00 & 0.00 & 54.76 & 2.72 \\
\hline Rhododendron ferrugineum* & Ericaceae & com & SHFO & 2 & 2 & $\operatorname{ccs}$ & 0.027 & 38.40 & 3.71 & & \\
\hline Rhododendron hirsutum* & Ericaceae & com & SHFO & 2 & 4 & $\operatorname{ccs}$ & 0.029 & 38.40 & 4.31 & & \\
\hline $\begin{array}{l}\text { Rhodothamnus } \\
\text { chamaecistus } *\end{array}$ & Ericaceae & sca & SHFO & 2 & 4 & $\operatorname{ccs}$ & 0.040 & 0.00 & 0.00 & 84.00 & 7.04 \\
\hline Salix helvetica* & Salicaceae & sca & SHFO & 1.5 & 2 & $\operatorname{ccs}$ & n.a & 0.00 & 0.00 & & \\
\hline Salix pentandra* & Salicaceae & rar & SHFO & 2.5 & 3 & $\mathrm{ccc}$ & 0.096 & 91.20 & 2.94 & & \\
\hline Saponaria pumila & Caryophyllaceae & com & GRASS & 1 & 2 & $\operatorname{css}$ & 1.210 & 0.00 & 0.00 & 10.16 & 2.59 \\
\hline Saussurea pygmaea & Asteraceae & $\operatorname{rar}$ & GRASS & 1 & 4 & $\operatorname{css}$ & 4.750 & 95.20 & 2.33 & & \\
\hline Saxifraga adscendens & Saxifragaceae & rar & ROSC & 1.5 & 4 & rss & 0.011 & 39.00 & 2.52 & & \\
\hline Saxifraga aizoides & Saxifragaceae & com & WET & 2 & 4 & $\operatorname{css}$ & 0.054 & 83.00 & 1.91 & & \\
\hline Saxifraga androsacea & Saxifragaceae & sca & ROSC & 1 & 4 & sss & 0.036 & 0.00 & 0.00 & 25.00 & 4.50 \\
\hline Saxifraga aspera & Saxifragaceae & sca & ROSC & 2 & 2 & $\operatorname{css}$ & 0.015 & 0.00 & 0.00 & 0.00 & 0.00 \\
\hline Saxifraga biflora & Saxifragaceae & sca & ROSC & 1 & 4 & $\operatorname{css}$ & 0.016 & 11.33 & 7.33 & 63.53 & 6.70 \\
\hline Saxifraga bryoides & Saxifragaceae & com & ROSC & 1 & 2 & $\operatorname{css}$ & 0.042 & 0.00 & 0.00 & 2.17 & 2.17 \\
\hline Saxifraga burseriana & Saxifragaceae & rar & ROSC & 2 & 5 & sss & 0.052 & 0.00 & 0.00 & 33.03 & 2.97 \\
\hline
\end{tabular}


Table 1 continued

\begin{tabular}{|c|c|c|c|c|c|c|c|c|c|c|c|}
\hline Species & Family & $\begin{array}{l}\text { Rar- } \\
\text { ity }\end{array}$ & $\begin{array}{l}\text { Commun- } \\
\text { ity }\end{array}$ & $\mathrm{T}$ & $\mathrm{R}$ & CSR & $\begin{array}{l}\text { Seed } \\
\text { mass }\end{array}$ & $\begin{array}{l}\text { FGP } \\
(\%)\end{array}$ & $\begin{array}{l}\text { SE } \\
\text { FGP }\end{array}$ & $\begin{array}{l}\text { GA } \\
\text { FGP }(\%)\end{array}$ & $\begin{array}{l}\text { SE } \\
\text { GA }\end{array}$ \\
\hline Saxifraga caesia & Saxifragaceae & com & GRASS & 1.5 & 5 & sss & 0.024 & 14.00 & 2.58 & 55.60 & 6.58 \\
\hline Saxifraga exarata & Saxifragaceae & rar & ROSC & 1 & 2 & sss & 0.055 & 0.00 & 0.00 & 4.00 & 1.63 \\
\hline Saxifraga hostii & Saxifragaceae & end & GRASS & 2 & 5 & sss & 0.055 & 0.00 & 0.00 & 12.87 & 4.04 \\
\hline Saxifraga moschata & Saxifragaceae & com & ROSC & 1 & 4 & sss & 0.040 & 22.00 & 4.16 & & \\
\hline Saxifraga mutata & Saxifragaceae & sca & ROSC & 2.5 & 4 & sss & 0.030 & 38.00 & 7.75 & & \\
\hline Saxifraga oppositifolia & Saxifragaceae & com & ROSC & 1 & 4 & css & 0.014 & 0.00 & 0.00 & 29.33 & 3.71 \\
\hline Saxifraga paniculata & Saxifragaceae & com & ROSC & 2 & 4 & sss & 0.040 & 0.00 & 0.00 & 19.35 & 2.89 \\
\hline Saxifraga rotundifolia & Saxifragaceae & sca & TALL & 2.5 & 3 & $\operatorname{ccs}$ & 0.039 & 96.00 & 2.83 & & \\
\hline Saxifraga rudolphiana & Saxifragaceae & end & ROSC & 1 & 4 & $\operatorname{css}$ & 0.070 & 80.00 & 1.63 & & \\
\hline Saxifraga sedoides & Saxifragaceae & sca & ROSC & 1.5 & 5 & $\operatorname{css}$ & 0.077 & 0.00 & 0.00 & 15.13 & 1.85 \\
\hline Saxifraga seguieri & Saxifragaceae & rar & ROSC & 1 & 2 & $\operatorname{css}$ & 0.037 & 1.00 & 1.00 & 47.36 & 4.37 \\
\hline Saxifraga squarrosa & Saxifragaceae & end & ROSC & 2 & 5 & sss & 0.029 & 29.00 & 8.70 & & \\
\hline Saxifraga stellaris & Saxifragaceae & com & WET & 2 & 3 & $\operatorname{css}$ & 0.040 & 93.00 & 3.42 & & \\
\hline Saxifraga tridactylites & Saxifragaceae & $\operatorname{rar}$ & DRYM & 4.5 & 4 & rrs & 0.011 & 0.00 & 0.00 & 54.48 & 10.11 \\
\hline Scabiosa lucida* & Dipsacaceae & sca & GRASS & 1.5 & 4 & crs & 1.841 & 52.80 & 6.37 & & \\
\hline Scorzonera humilis & Asteraceae & sca & WET & 3.5 & 4 & $\operatorname{ccs}$ & 4.068 & 3.20 & 1.50 & & \\
\hline Scorzoneroides crocea & Asteraceae & rar & MEAD & 2 & 3 & $\mathrm{crs}$ & 2.198 & 51.20 & 4.27 & & \\
\hline Scorzoneroides helvetica & Asteraceae & com & MEAD & 1.5 & 2 & crs & 1.570 & 73.60 & 2.04 & & \\
\hline Sedum album & Crassulaceae & com & ROSC & 3 & 4 & sss & 0.040 & 72.00 & 5.51 & & \\
\hline $\begin{array}{l}\text { Senecio abrotanifolius ssp. } \\
\text { abrotanifolius* }\end{array}$ & Asteraceae & sca & SHFO & 2 & 4 & $\operatorname{css}$ & 1.989 & 53.60 & 8.16 & & \\
\hline $\begin{array}{l}\text { Senecio abrotanifolius ssp. } \\
\text { tiroliensis }\end{array}$ & Asteraceae & sca & SHFO & 1.5 & 2 & $\operatorname{css}$ & n.a & 20.80 & 2.65 & & \\
\hline Senecio doronicum & Asteraceae & sca & GRASS & 1.5 & 4 & $\operatorname{css}$ & 1.800 & 13.60 & 4.49 & 65.34 & 6.80 \\
\hline Seseli libanotis & Apiaceae & sca & DRYM & 3 & 4 & $\operatorname{ccs}$ & 1.309 & 8.80 & 3.20 & 28.80 & 2.94 \\
\hline Sesleria ovata & Poaceae & sca & ROSC & 1 & 5 & $\operatorname{css}$ & 0.008 & 0.00 & 0.00 & & \\
\hline Silene acaulis ssp. exscapa & Caryophyllaceae & sca & GRASS & 1 & 2 & $\operatorname{css}$ & 0.419 & 32.00 & 6.57 & & \\
\hline $\begin{array}{l}\text { Silene acaulis ssp. } \\
\text { longiscapa* }\end{array}$ & Caryophyllaceae & com & GRASS & 1 & 4 & $\operatorname{css}$ & 0.379 & 39.20 & 4.80 & & \\
\hline Silene nutans & Caryophyllaceae & com & SHFO & 3 & 3 & $\operatorname{ccs}$ & 0.368 & 69.60 & 6.01 & & \\
\hline Silene vulgaris* & Caryophyllaceae & com & MEAD & 3 & 3 & $\mathrm{crs}$ & 1.535 & 81.60 & 9.17 & & \\
\hline Soldanella alpina & Primulaceae & com & SHFO & 2 & 3 & $\operatorname{css}$ & 0.240 & 83.20 & 1.50 & & \\
\hline Soldanella pusilla* & Primulaceae & com & GRASS & 1.5 & 2 & $\operatorname{css}$ & 0.069 & 84.00 & 2.83 & & \\
\hline Solidago virgaurea* & Asteraceae & com & SHFO & 3.5 & 3 & $\mathrm{crs}$ & 0.690 & 75.86 & 4.96 & & \\
\hline Tofieldia calyculata* & Tofieldiaceae & sca & WET & 2.5 & 4 & $\operatorname{css}$ & 0.030 & 74.62 & 6.26 & & \\
\hline Tofieldia pusilla* & Tofieldiaceae & rar & WET & 1.5 & 3 & $\operatorname{css}$ & 0.035 & 92.80 & 2.33 & & \\
\hline Trichophorum alpinum & Cyperaceae & rar & WET & 3 & 2 & $\operatorname{css}$ & 0.182 & 0.00 & 0.00 & 1.67 & 3.73 \\
\hline Trichophorum cespitosum* & Cyperaceae & sca & WET & 2.5 & 3 & $\operatorname{css}$ & 0.584 & 0.00 & 0.00 & 0.00 & 0.00 \\
\hline Trifolium badium & Fabaceae & sca & GRASS & 2 & 4 & $\mathrm{crs}$ & 0.750 & 55.20 & 3.44 & & \\
\hline Triglochin palustris* & Juncaginaceae & sca & WET & 2.5 & 4 & sss & 1.761 & 54.40 & 5.60 & & \\
\hline Trollius europaeus & Ranunculaceae & sca & MEAD & 2.5 & 3 & $\operatorname{ccs}$ & 0.810 & 0.80 & 0.80 & 41.50 & 2.01 \\
\hline Valeriana celtica* & Valerianaceae & end & GRASS & 1 & 2 & $\operatorname{css}$ & 0.675 & 0.00 & 0.00 & & \\
\hline Valeriana elongata* & Valerianaceae & end & ROSC & 2 & 5 & $\mathrm{crs}$ & n.a & 9.60 & 2.40 & & \\
\hline
\end{tabular}


Table 1 continued

\begin{tabular}{|c|c|c|c|c|c|c|c|c|c|c|c|}
\hline Species & Family & $\begin{array}{l}\text { Rar- } \\
\text { ity }\end{array}$ & $\begin{array}{l}\text { Commun- } \\
\text { ity }\end{array}$ & $\mathrm{T}$ & $\mathrm{R}$ & CSR & $\begin{array}{l}\text { Seed } \\
\text { mass }\end{array}$ & $\begin{array}{l}\text { FGP } \\
(\%)\end{array}$ & $\begin{array}{l}\text { SE } \\
\text { FGP }\end{array}$ & $\begin{array}{l}\text { GA } \\
\text { FGP }(\%)\end{array}$ & $\begin{array}{l}\text { SE } \\
\text { GA }\end{array}$ \\
\hline Valeriana montana & Valerianaceae & com & ROSC & 2 & 5 & $\operatorname{css}$ & 1.103 & 4.80 & 2.94 & 35.80 & 6.53 \\
\hline Valeriana supina & Valerianaceae & end & ROSC & 1 & 5 & $\operatorname{css}$ & 1.232 & 3.20 & 1.50 & & \\
\hline Valeriana tripteris & Valerianaceae & com & TALL & 2.5 & 3 & $\operatorname{css}$ & 0.926 & 7.20 & 2.33 & 31.10 & 5.03 \\
\hline Veratrum album & Melanthiaceae & sca & TALL & 2.5 & 4 & $\operatorname{ccs}$ & 7.060 & 0.00 & 0.00 & 3.08 & 1.28 \\
\hline Veronica alpina & Antirrhinaceae & com & GRASS & 1.5 & 2 & $\operatorname{css}$ & 0.040 & 80.00 & 5.66 & & \\
\hline Veronica aphylla & Antirrhinaceae & com & GRASS & 1.5 & 5 & $\operatorname{css}$ & 0.099 & 0.00 & 0.00 & 3.20 & 2.33 \\
\hline Veronica bellidioides* & Antirrhinaceae & sca & GRASS & 1 & 1 & $\operatorname{css}$ & 0.110 & 0.80 & 0.80 & 68.46 & 4.63 \\
\hline Veronica fruticans & Antirrhinaceae & sca & GRASS & 1.5 & 3 & $\operatorname{css}$ & 0.124 & 1.80 & 0.98 & 4.06 & 2.19 \\
\hline
\end{tabular}

Community affiliation, temperature value (T), soil reaction value (R) and CSR strategy type were extracted from Landolt et al. (2010) and grouped as described in Material and Methods. Seed mass (mg/seed) was determined by seed collectors or extracted from data bases. Germination was tested in a growth cabinet using a standard protocol with one alternating temperature $25 / 15{ }^{\circ} \mathrm{C}$ or, if marked with asterisks $(*)$ with $20 / 10{ }^{\circ} \mathrm{C}$. Final germination percentage (FGP \%) is calculated as germinated seeds from the initial number of seeds laid per species (see Supplementary Material Table S1). If FGP was below 15\%, a treatment with gibberellic acid (GA) was applied

Elevation values (T) 1-1.5 = nival-alpin, 2-2.5 = subalpine, $3-4.5=$ montane-colline. Bedrock values $(\mathrm{R}) 1=$ acidic, $2-3=$ acidicneutral, 4-5 = neutral-alkaline. Stress tolerant (S) = SSS, CSS, RSS; competitive (C) = CCC, CCS, CCR; intermediate strategists $\mathrm{CSR}$; ruderal $(\mathrm{R})=\mathrm{RRR}, \mathrm{RRS}, \mathrm{RRC}$

com common, sca scattered, rar rare, end endemic, ROSC rocky habitats, scree sites, GRASS alpine grasslands above treeline, WET bogs, mires, wetlands, SHFO scrub heaths, understorey of forests, subalpine meadows, TALL tall forbs, DRYM dry meadows of low elevations, $M E A D$ meadows of the colline and montane belt, n.a. not available, $S E$ Standard error

\section{Germination protocol}

The germination tests were carried out at the laboratory in Innsbruck, following a standard protocol. Tests were done on Petri dishes filled with three layers of filter paper moistened with deionized water. In each Petri dish, 25 seeds were laid and five Petri dishes per species were prepared. Depending on seed availability, either the number of seeds laid per Petri dish or the number of Petri dishes was adjusted (total numbers of seed laid are given in Supplementary Material Table S1). The Petri dishes were then put in a growth cabinet (SANYO MLR-350H, Sanyo Electric Biomedical Co Ltd, Japan) under $16 \mathrm{~h}$ day $(20,0001 \times)$ and $8 \mathrm{~h}$ night $(01 \times)$ regime with $60 \%$ relative air humidity. The maximum photosynthetic photon flux density in the growth cabinet was $180 \mu \mathrm{mol} \mathrm{m} \mathrm{m}^{-2} \mathrm{~s}^{-1}$. Initially, a day/night temperature regime of $20 / 10{ }^{\circ} \mathrm{C}$ was used (see species with $*$ in Table 1) according to former germination protocols for alpine species (Schwienbacher and Erschbamer 2001; Schwienbacher et al. 2012; Walder and Erschbamer 2015). Later, the setting was changed to a day/night temperature regime of $25 / 15^{\circ} \mathrm{C}$ according to the protocol of ENSCONET (2009b). Due to former experiences with alpine species (Schwienbacher and Erschbamer 2001; Erschbamer and Pfattner 2002) and due to technical reasons, we did not stratify the seeds. The only exceptions were Saxifraga species; for them we applied cold-wet stratification in the fridge at $+4{ }^{\circ} \mathrm{C}$ for 12 weeks.

The number of germinated seeds was counted every 3-4 days and germinated seeds (radicle emergence at least half as long as the seed) were removed. Seeds infested with fungi were subjected to a pressure test using tweezers and, in the case of softness, removed from the Petri dish and counted as non-viable. Using the same method, non-germinated seeds were checked for vitality at the end of the test period and were counted as non-viable if not passing the test. One germination period lasted for 30 days. The final germination percentage (FGP) after 30 days was calculated as germinated seeds from the initial number of seeds laid, pooled from all Petri dishes, representing a mean per species.

Seeds of species with no or $\leq 15 \%$ germination were soaked in gibberellic acid (0.001 molar $\mathrm{GA}_{3}$, $10 \mathrm{ml}$ ) for 3 days in the growth cabinet to stimulate 
their germination, assuming non-deep physiological dormancy (Baskin and Baskin 2014). After 3 days, seeds were washed with deionized water and were again laid out on Petri dishes with three layers of filter paper in the growth cabinet. Final germination percentage after $\mathrm{GA}_{3}$ treatment (GA FGP) was determined after another 30 days. For species of the families Cyperaceae and Apiaceae from the 2018 collection, parallel $\mathrm{GA}_{3}$ treatment to the standard tests were performed due to the availability of enough seeds. For 10 species with zero or $\leq 15 \%$ FGP, $\mathrm{GA}_{3}$ treatment was not possible due to scarcity of seeds. Numbers of seeds laid, germinated seeds and nonviable seeds are given in Supplementary Material Table S1.

\section{Classifications}

Similarly to Alsos et al. (2013), species with FGP $\leq 10.0 \%$ were classified as very weak germinators, FGP of $10.1-20.0 \%$ as low germinators, $20.1-50.0 \%$ as intermediate ones and those with $50.1-100 \%$ as species with high germination. Species rarity was extracted from Fischer et al. (2008) and the following classes were defined: com (common, species occurring very frequently in the Eastern Alps); sca (scattered, species not very frequent, but in some regions of the Eastern Alps they may be locally common); rar (rare); end (endemic for the North-Eastern or South Alps). A classification of the test species addressing their ecology was done using ecological indicator values (i.e. temperature (T), soil reaction (R), CSR strategy) and affiliation to plant communities provided by (Landolt et al. 2010). The test species were grouped in seven plant communities: ROSC = rocky habitats, scree sites; GRASS = alpine grasslands above treeline; $\mathrm{WET}=$ bogs, mires, wetlands; $\mathrm{SHFO}=$ scrub heaths, understorey of forests, subalpine meadows; TALL $=$ tall forbs; DRYM = dry meadows of low elevations; $\mathrm{MEAD}=$ meadows of the colline and montane belt. Temperature values $(\mathrm{T})$ act as a proxy for the elevation distribution of a species, they were grouped as follows: 1-1.5 = niv-alp (nival, upper alpine belt, lower alpine belt); 2-2.5 = subalpine (subalpine belt, upper montane belt); 3-4.5 = moncoll (montane belt, colline belt). Ranges of soil reaction $(\mathrm{R})$ represent the bedrock on which the species mostly occurs. These values were distinguished as: 1 = acidic (very acidic and acidic);
2-3 = aci-neu (weakly acidic, neutral to alkaline); 4-5 = neu-alk (alkaline). CSR strategies were grouped in $\mathrm{S}=$ stress tolerant (SSS, CSS, RSS); $\mathrm{C}=$ competitive (CCC, CCS, CCR); CSR = intermediate strategists; $\mathrm{R}=$ ruderal (RRR, RRS, RRC). Further, seed mass (mg/seed) was either determined by seed collectors, or extracted from the following databases: KEW Seed Information Database (SID), The LEDA Traitbase, BiolFlor Database, The VISTA Plant Trait Database, Ecological Flora of the British Isles.

\section{Data analyses}

We defined FGP $\geq 20.1 \%$ (i.e. species with intermediate and high germination) as successful proof of the functioning of the standard protocol. For species with FGP $\leq 20.0 \%$, we assumed that the standard protocol did not work and therefore excluded these species from further analyses. In addition, the strategy type R was excluded from the analyses, because this group contained only one species with FGP $\geq 20.1 \%$ (Centaurium erythraea). Using this reduced dataset, FGP (species means) was analysed by a generalized linear model (GLM) with a quasi-binomial setting and a logit-link function. A binomial distribution is typically used for proportion data such as germinated seeds from seeds laid. The model showed an over-dispersion. Therefore, a quasi-binomial setting was used. This setting accounts for over-dispersion by means of a dispersion parameter that allows additional variance in the data and returns adjusted standard errors.

The full model included all factors, i.e. rarity (sca, com, rar, end), plant community (ROSC, GRASS, WET, SHFO, TALL, DRYM, MEAD), elevation belt (niv-alp, subalpine, mon-coll), bedrock type (acidic, aci-neu, neu-alk), strategy type (S, C, CSR) and seed mass. F-test was used to test the significance of the factors in the model. Post hoc tests with BonferroniHolm correction were applied to test for significances between the levels of the factors. The significance level was set to alpha 0.05 ; the model was verified via diagnostic residual plots. Because only one factor showed significance, the factors were ranked according to least significance and were successively excluded from the model to check the influence of the factors on the model. This procedure was done to prevent an over-fitting of the model, but as this did not lead to any changes in factor level differences 
compared to the full model, the full model was chosen as the final one.

FGP for species from the families Asteraceae, Poaceae, and Saxifragaceae are visually shown to highlight the germination behaviour of these families.

The analyses were performed in $\mathrm{R}$ (version 3.6.1, $\mathrm{R}$ Core Team 2020) and RStudio (version 1.2.1568, RStudio 2016) with the MASS package (Venables and Ripley 2002). For data manipulation, we used the packages tidyverse (Wickham 2017) and reshape (Wickham 2018a), for descriptive statistics the package pastecs (Grosjean et al. 2018). For data visualization, we used the packages ggplot2 (Wickham and Winston 2019), gridExtra (Auguie and Antonov 2017) and scales (Wickham 2018b).

\section{Results}

In total, 255 species out of 39 families were tested. Regarding the germination classes, $74.9 \%$ germinated under the standard protocol, and $25.1 \%$ failed to germinate (Table 1, Fig. 1a). From the 255 species, $31.4 \%$ had a high FGP. Only one species among the tested (Carlina acaulis) showed 100\% FGP. Intermediate germination was observed in $17.6 \%$, low germination was noted for $6.3 \%$, and $19.6 \%$ had a weak germination. Scattered species represented about half of the species (48.6\%, Fig. 1b), being the largest group in this class. Species from alpine grasslands represented $35.7 \%$ of the species tested (Fig. 1c); $43.9 \%$ were classified as species from the niv-alp elevation belt (Fig. 1d). Of all species tested, only $2.8 \%$ were classified as species on very acidic bedrock (Fig. 1e), while the classes aci-neu and neu-alk contained $47.1 \%$ and $50.2 \%$ of species, respectively. Half of the species tested $(50.6 \%)$ was classified as being stress tolerant (S, Fig. 1f), and only $1.6 \%$ represented the ruderal strategy type (R). Seed mass ranged from 0.001 to $9.68 \mathrm{mg} / \mathrm{seed}$, with Dactylorhiza incarnata having the lightest seeds and Laserpitium latifolium the heaviest.

The mean FGP was analysed by a GLM using a dataset of 124 species, comprising species with FGP $\geq 20.1 \%$. Strategy type was the only significant factor in the model (Table 2). However, post hoc tests did not reveal any significances between the strategy type factor levels. We could not find any statistically significant effects of rarity, plant community, (a)

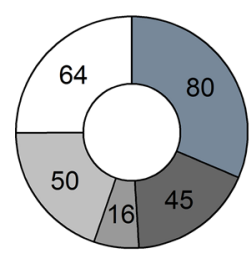

(d)
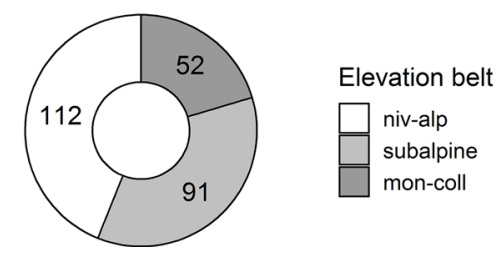

(b)
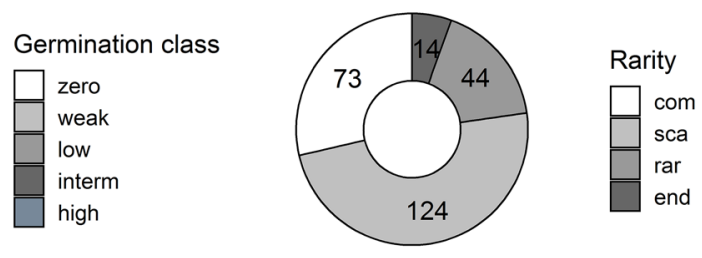

(e)

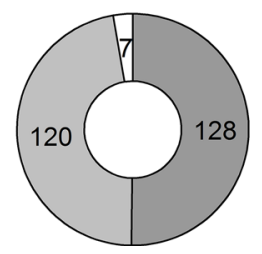

(c)

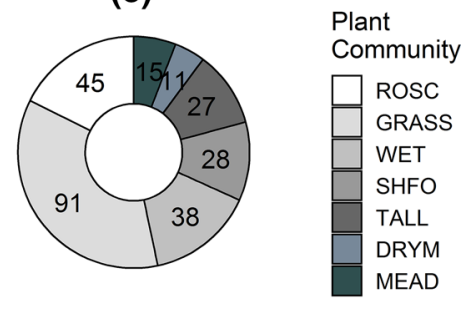

(f)

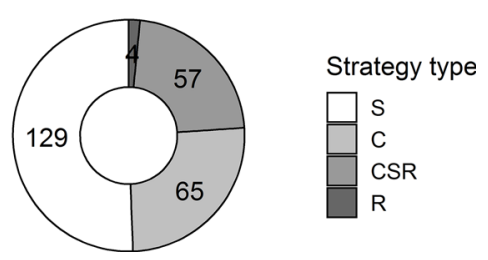

Fig. 1 Species tested using the standard protocol (total 255 species) and classifications made in the study. The graphs present the numbers of species per class: a Germination class defined as zero: $\mathrm{FGP}=0.0 \%$, weak: $\mathrm{FGP} \leq 10.0 \%$, low: FGP $\geq 10.1 \leq 20.0 \%$, intermediate: $F G P \geq 20.1 \leq 50.0 \%$ and high: FGP $\geq 50.1-100.0 \%$. b Rarity (com common, scat scattered, rar rare, end endemic). c Plant community (ROSC rocky habitats, scree sites, GRASS alpine grasslands above treeline, WET bogs, mires, wetlands, SHFO scrub heaths, understorey of forests, subalpine meadows, TALL tall forbs, $D R Y M$ dry meadows of low elevations, MEAD meadows of the colline and montane belt). d Elevation belt (nival, subalpine, montane-colline). e Bedrock type (acidic, acidic-neutral, acidicalkaline), and $\mathbf{f}$ Strategy type $S$ stress tolerant, $C$ competitive, $C S R$ intermediate, $R$ ruderal strategy 
Table 2 Analyses of deviance-table listing the effects of tested factors on species mean FGP, comprising species with FGP $\geq 20.1 \%$

\begin{tabular}{llllll}
\hline Factors & Df & Deviance & $F$ value & $p$ value & Label \\
\hline Rarity & 3 & 16.604 & 0.816 & 0.488 & n.s \\
Plant community & 6 & 56.765 & 1.395 & 0.224 & n.s \\
Elevation belt & 2 & 7.374 & 0.544 & 0.582 & n.s \\
Bedrock type & 2 & 15.817 & 1.166 & 0.316 & n.s \\
Strategy type & 3 & 42.312 & 3.119 & 0.049 & $*$ \\
Seed mass & 1 & 3.201 & 0.472 & 0.494 & n.s \\
\hline
\end{tabular}

A GLM with quasi-binomial error and logit-link function was used to analyse the data; F-test was used to test the significance of the factors in the model

Dispersion parameter in the GLM was taken to be 6.8

$D f$ degrees of freedom; n.s. not statistically significant

Significance levels are labelled as. $p \sim 0.05,{ }^{*} p<0.05$, $* * p<0.01$, *** $p<0.001$

elevation belt, bedrock type or seed mass on FGP. Consequently, post hoc tests on the single factors per class resulted in non-significant differences. Common and endemic species had the lowest mean FGP (55.4\% and $55.8 \%$, respectively), and rare species the highest (67.7\%; Fig. 2a). Within plant communities (Fig. 2b), the lowest FGP was found in GRASS species (54.7\%), the highest in the WET community class $(72.5 \%)$. In contrast to this high FGP, the WET class had the highest proportion of non-germinating, weak and low germinating species (i.e. 23 out of 38 species, Fig. 1c and Fig. 2b). Species from the mon-coll elevation belt had the highest mean FGP (67.4\%) followed by subalpine species $(60.5 \%)$ and niv-alp species (58.5\%; Fig. 2c). Species classified to the acidic bedrock type had the lowest FGP (52.5\%), neu-alk the highest (62.5\%, Fig. 2d). Regarding strategy types (Fig. 1e), $\mathrm{C}$ strategists had the lowest $(56.7 \%)$ and intermediate strategists the highest FGP (65.2\%, Fig. 2e). Seed mass (Fig. 2f) of species with FGP $\geq 20.1 \%$ ranged from $0.01 \mathrm{mg} / \mathrm{seed}$ (Saxifraga adscendens) to $5.40 \mathrm{mg} / \mathrm{seed}$ (Cirsium carniolicum).

A treatment with gibberellic acid $\left(\mathrm{GA}_{3}\right)$ was applied to $44.7 \%$ out of 255 species (Fig. 3). Germination was stimulated in half of them $(50.8 \%$ out of 114 species), resulting in GA FGPs of 20.1-97.6\%. The other half of the $\mathrm{GA}_{3}$ tested species comprised $29.8 \%$, classified as low and weak germinators, and $19.3 \%$ did not germinate (Fig. 3). Illustrations of GA FGP of the species according to the classifications can be found in the Supplementary Material Fig. S1.
The most abundant species collected were from the family Asteraceae (53 species, Fig. 4), whereby $86.8 \%$ had FGP $\geq 20.1-100 \%$, and $13.2 \%$ had low and weak germination. None of the Asteraceae species was noted to have zero germination using the standard protocol. Poaceae species (17 species) germinated with a similar pattern to Asteraceae species (Fig. 5). $58.8 \%$ had high to intermediate germination, $35.3 \%$ had low and weak germination, and only one species did not germinate under the standard protocol (Sesleria ovata). Species among the family Saxifragaceae (21 species) were cold stratified before the germination test. Here, $38.1 \%$ had FGP $\geq 20.1 \%$, and $47.6 \%$ did not germinate under the standard protocol (Fig. 6a). On 13 species a $\mathrm{GA}_{3}$ treatment was applied (Fig. 6b), which enhanced the germination of 12 species, one did not germinate at all (Saxifraga aspera).

\section{Discussion}

In total, $74.9 \%$ of all investigated species germinated using the standard protocol, and among them, $49 \%$ had an intermediate to high FGP. If it is noted that half of the species germinate, this is a fairly good result, indicating that the collected seeds were in good condition and able to germinate without pre-treatment (except for the Saxifragaceae which were stratified). High-quality seeds are mandatory for ex situ seed banking. Alsos et al. (2013) suggested to consider the annual variation of seed production and to extend seed 
(a) Rarity

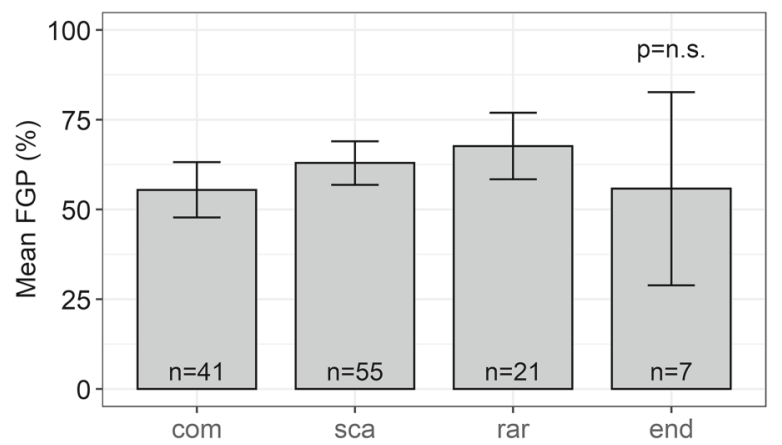

(c) Elevation belt

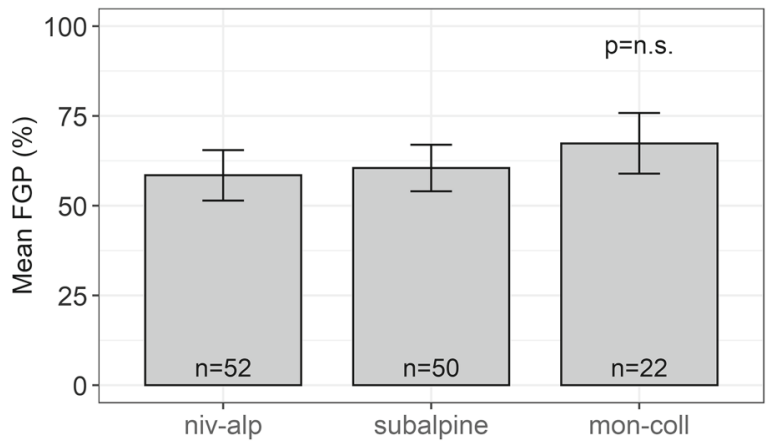

(e) Strategy type

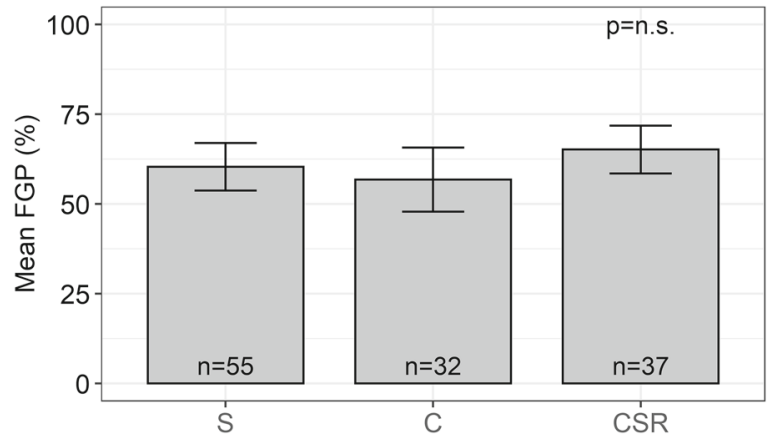

Fig. 2 Final germination percentage (FGP) of intermediate and high germinating species (in total 124 species), tested using the standard protocol and analysed via a GLM. Comparisons between the levels of the classes a Rarity, b Plant community, c Elevation belt, $\mathbf{d}$ Bedrock type and $\mathbf{f}$ Strategy type were made with Post hoc tests and Bonferroni-Holm correction. For

collections over several seasons to overcome possible insufficient germination from one season. This would further increase the genetic diversity of the stored seeds. From our field collections, we have learned that several rare and endemic species often produce seeds in small quantities and over such a long ripening

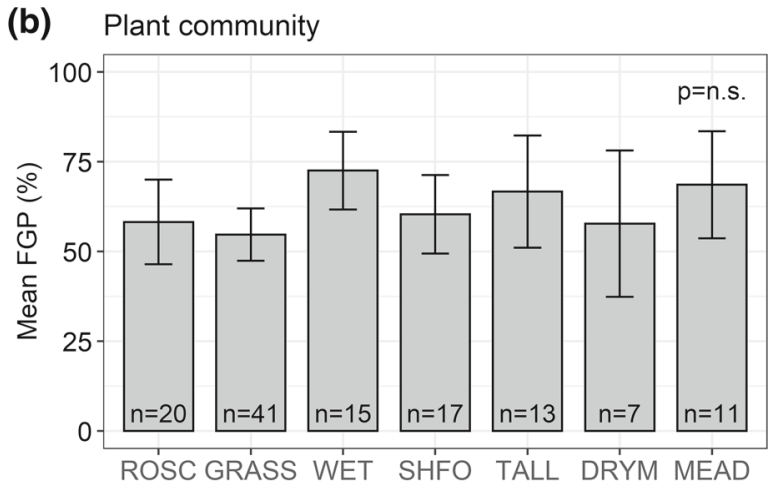

(d) Bedrock type

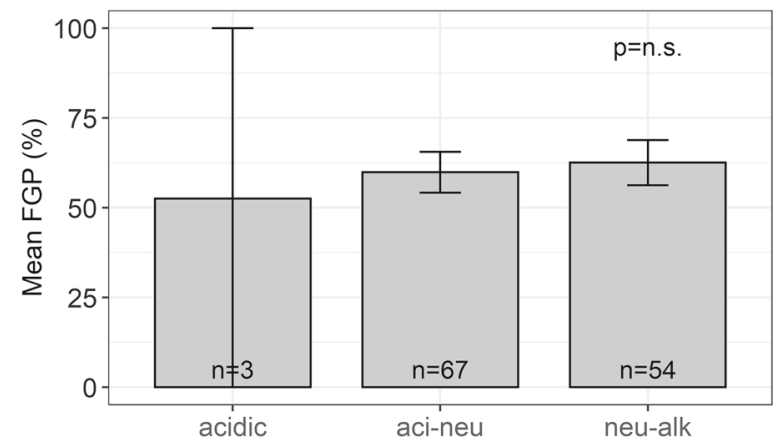

(f) Seed mass

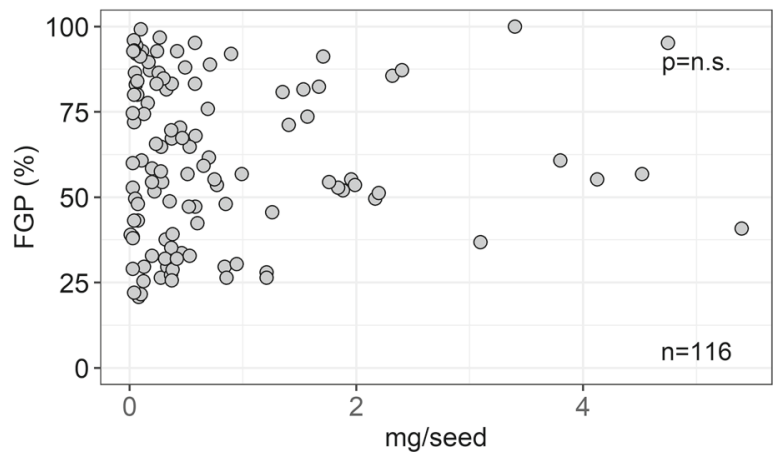

abbreviations see Fig. 1 or Material and Methods. $n$ number of species per class. In e Seed mass was not available for 8 species. Error bars show confidence intervals $(95 \%)$. $p$ indicates the $p$ value label from the post hoc tests, n.s. not statistically significant

period that the necessary amount of seeds for seed banking and germination trials cannot be obtained within one year. A complete ex situ seed bank of rare and endemic species seems therefore hardly possible. Nonetheless, seed banks make an important contribution to the conservation of these species, but strict 


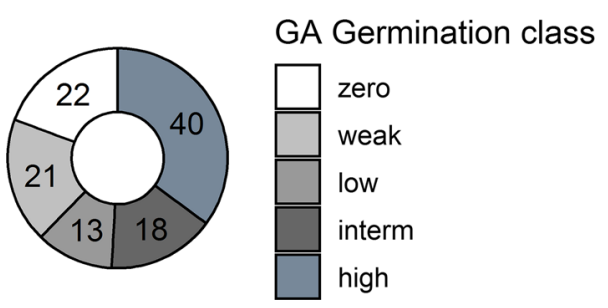

Fig. 3 Numbers of species tested with a gibberellic acid treatment (GA) using the standard protocol (total 114 species), and grouped in the germination classes zero: FGP $=0.0 \%$, weak: FGP $\leq 10.0 \%$, low: FGP $\geq 10.1 \leq 20.0 \%$, intermediate: FGP $\geq 20.1 \leq 50.0 \%$ and high: FGP $\geq 50.1-100.0 \%$

conservation measures in the field are indispensable, including the protection of entire plant communities.

The standard protocol used in this study with only one temperature regime, worked well in a high percentage of species, but $25.1 \%$ did not germinate. This amount of non-germinating species weakens an ecological interpretation of the results regarding germination success. It seems to be mandatory to perform germination trials at least under two (or preferably more) different temperature regimes to fully cover the germination niche of a species, or to identify unknown germination needs. In addition, seed pre-treatments, such as cold-wet stratification and scarification, would have been necessary for at least some of the species studied. For technical reasons, many species have been tested after a relatively long storage period (dry-cold). In some of these cases, storage may have induced seed dormancy (Baskin and Baskin 2014). Considering these limitations of the study, we must interpret our results with caution.

(a) A classification of the tested species into common, scattered, rare and endemic species did not reveal differences of FGP. Despite the uneven quantity of species within these classes, about $50 \%$ of the species in each class germinated, and about $50 \%$ did not germinate. This may indicate that widespread species and species with a small distribution range do not have different germination temperature requirements, or these results may indicate that there are differences. For ex situ seed banking, these results could mean a 50/50 chance of germination success for rare and endemic Alpine species, when testing under a single standard protocol. Further, this strongly suggests applying more temperature regimes if seeds are available. In any case, besides germination in the laboratory, field experiments to analyse recruitment success under different environmental conditions are necessary, to test the performance and population persistence of these groups (Margreiter et al. in review).

(b) Although germination traits are important drivers of community composition and species persistence (Donohue et al. 2010; JiménezAlfaro et al. 2016; Tudela-Isanta et al. 2018a, b), no clear effect of habitat on germination was found in our study. The highest germination was recorded for species from WET environments (although several Carex species did not germinate, Table 1) and from MEAD. The conditions for seed development might have been favourable in these habitats, whereas in other habitats the growing seasons are often too short (ROSC, GRASS) or unfavourable for a complete seed maturation (DRYM, TALL, SHFO). This is in line with the findings of $\mathrm{Bu}$ et al. (2007) who germinated seeds of alpine species at the eastern Tsinghai-Tibet plateau, China, and in other habitats, when used germination temperatures that did not reflect the distribution areas of the tested species (Schütz et al. 1997; Thompson et al. 1999).

(c) Under the temperature regime used, there was no significant pattern of FGP between species from high and low elevations, but we may spot a trend with nival-alpine-subalpine species having lower FGPs compared to montane-colline centred species. In situ, a low germination may be the bottleneck for alpine seedling establishment under climate change (Shevtsova et al. 2009; Graae et al. 2011), since a high mortality of alpine seedlings was evidenced by several authors (Marcante et al. 2009; Graae et al. 2011; Winkler et al. 2015; Milbau et al. 2017). A high germination percentage could mean an important pre-requisite for a successful invasion of species from lower to higher elevations. Thus, novel communities can arise (Alexander et al. 2015, 2018), and it is unknown how coexistence or competition will shape species turnovers and community compositions in the future.

(d) The effect of bedrock (soil reaction) on germination has been notably investigated in the field (Forbis 2003; Wenk and Dawson 2007; Isselin- 


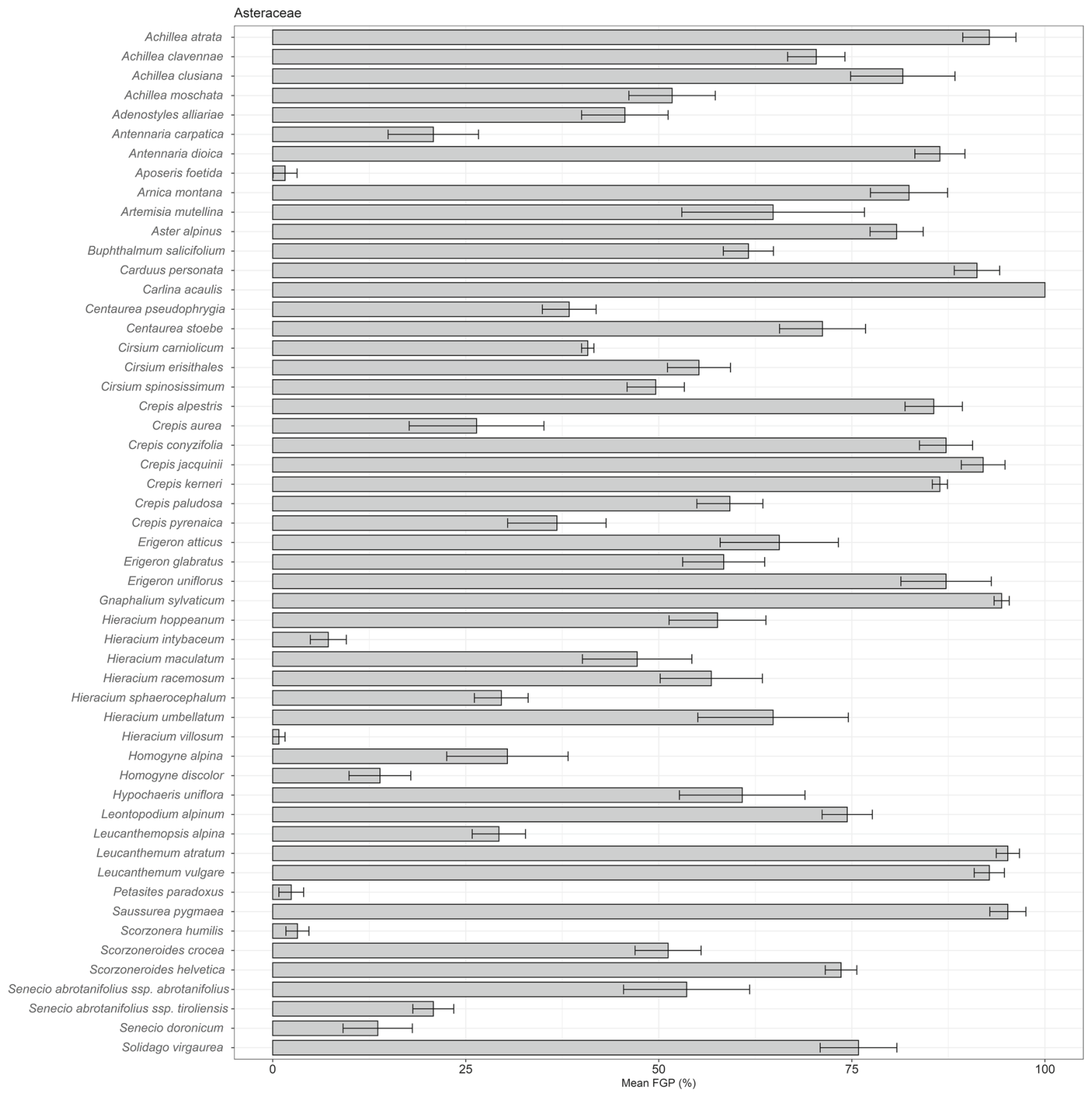

Fig. 4 Final germination percentages (FGP) of 53 species from the family Asteraceae, tested under the standard protocol. Error bars show standard errors

Nondedeu and Bédécarrats 2013), with the result, that soil chemistry was not related to FGP. Recent studies however (Fernández-Pascual et al. 2017; Tudela-Isanta et al. 2018a, b), reported contrasting results stating a consistent relation between germination strategy and bedrock type. Our results on FGP classified to three bedrock types, did not reveal any significant differences. However, we may note a trend of species from very acidic bedrock having a lower germination rate than species from slightly acidic to neutral soil reaction sites.

(e) Key parts of the CSR strategy concept (Grime 1979; Pierce et al. 2014) and the leaf-heightseed concept (LHS, Westoby 1998) are seed traits such as seed mass and volume, where seed mass is thought to be an indicator for seedling survival. Jiménez-Alfaro et al. (2016) 
Fig. 5 Final germination percentage (FGP) of 17 species from the family Poaceae, tested under the standard protocol. Error bars show standard errors

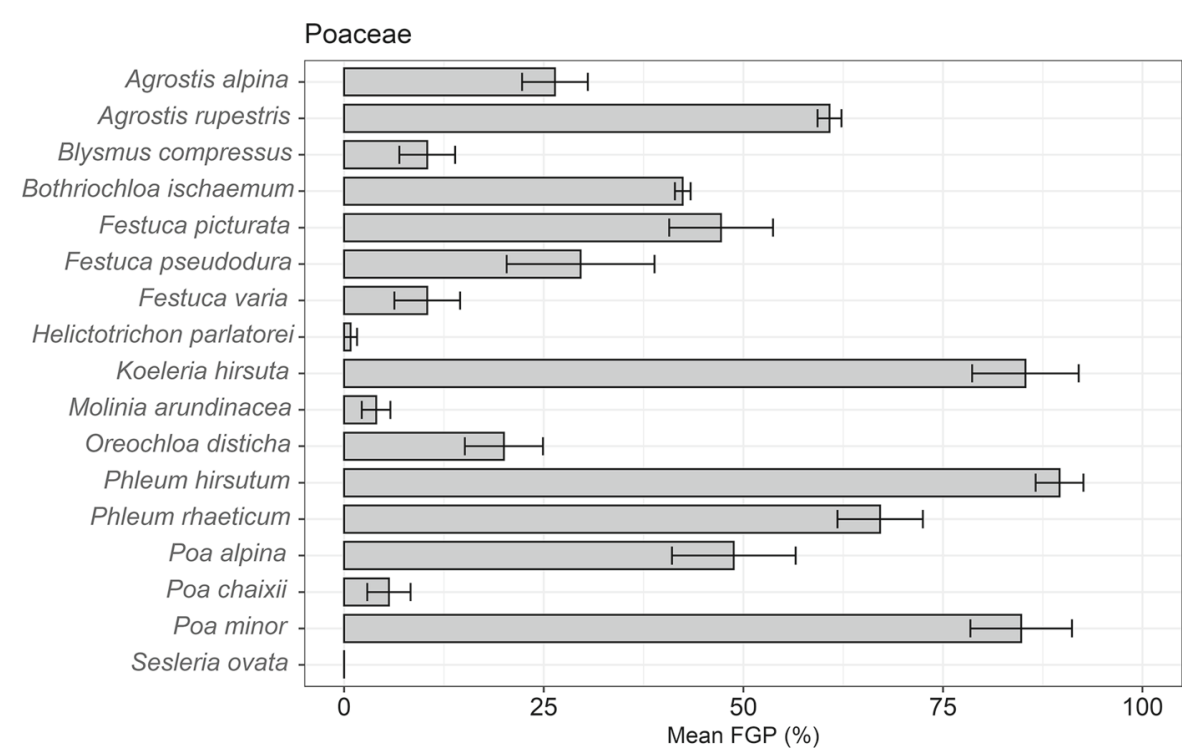

mentioned that germination does hardly correlate with CSR or LHS traits. This can be supported by our results, as no pattern was found. Stress tolerant species built the biggest group in alpine environments (Caccianiga et al. 2006) as also seen by our collections, and ruderal species occur only in a small proportion.

(f) No correlation was found between seed mass and FGP of species with high and intermediate germination. This is in line with results of a glacier foreland study, where representative species from different successional stages were investigated (Schwienbacher et al. 2012). In contrast, seed mass was highly related to FGP of selected glacier foreland species, after a winter burial trial in the field (Schwienbacher and Erschbamer 2001). Liu et al. (2013) reported of significant responses to temperature fluctuations of small seeded species compared to larger seeded ones. Generally, seed mass is thought to be an indicator for seedling survival (Grubb 1977; Westoby 1998; Coomes and Grubb 2003), and to correlate with several species traits (Fenner and Thompson 2005; Moles and Westoby 2006). Further, seed mass has also been regarded as an indicator for the competitive ability of a species (Tilman 1994). Thus, seed mass might be an essential trait for seedling performance, but not for FGP per se. (g) A treatment with $\mathrm{GA}_{3}$ was applied to $44.7 \%$ of the investigated species. In half of these species, germination was stimulated. They can be classified as having non-deep and intermediate physiological dormancy (Baskin and Baskin 2004, 2014). For the other half, deep physiological dormancy must be assumed. According to Schwienbacher et al. (2011), breaking deep physiological dormancy requires a cold-wet stratification for more than four months and additional preconditions such as temperature fluctuations (Baskin and Baskin 2014). Several authors suggested that cold-wet stratification is necessary for alpine species to germinate (Cavieres and Arroyo 2000; Shimono and Kudo 2003; García-Fernández et al. 2015; FernándezPascual et al. 2017), because this simulates a winter period which is an essential factor for inducing germination in spring. Cavieres and Sierra-Almeida (2018) recently found that this stratification effect does not increase with elevation. For some alpine species, even negative effects of stratification occur (GiménezBenavides and Milla 2013). Morphophysiological dormancy might have also occurred in the non-germinated species of our study, for instance in species from Apiaceae, Campanulaceae, Gentianaceae, and Ranunculaceae (Threadgill et al. 1981; Forbis and Diggle 2001; Baskin and Baskin 2005; Vandelook 
(a)

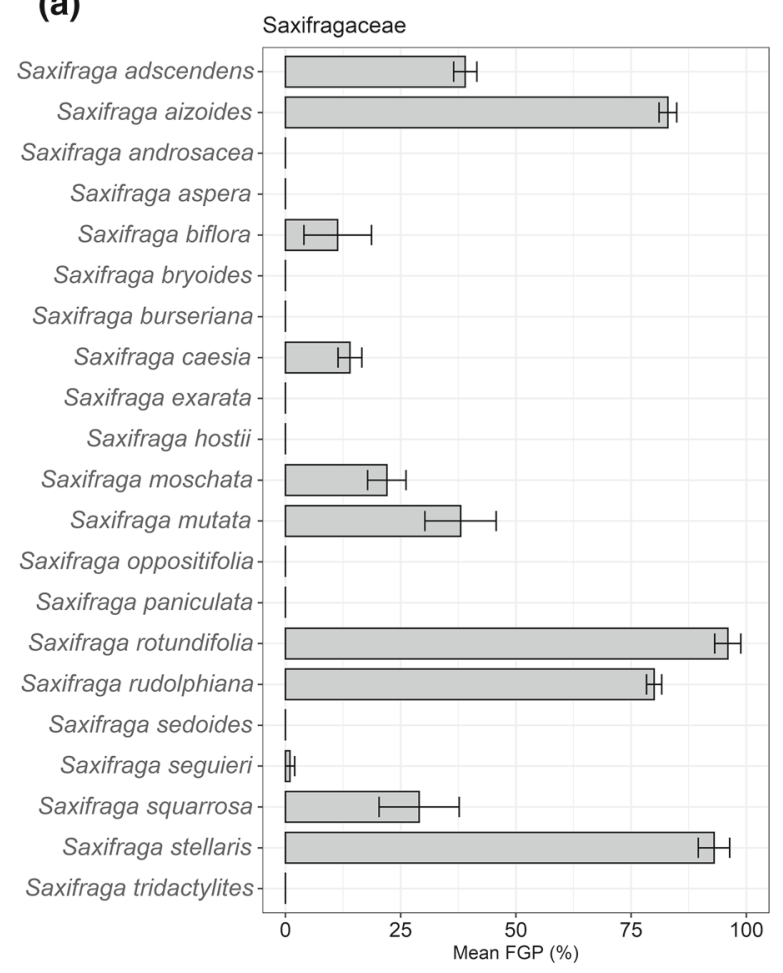

(b)

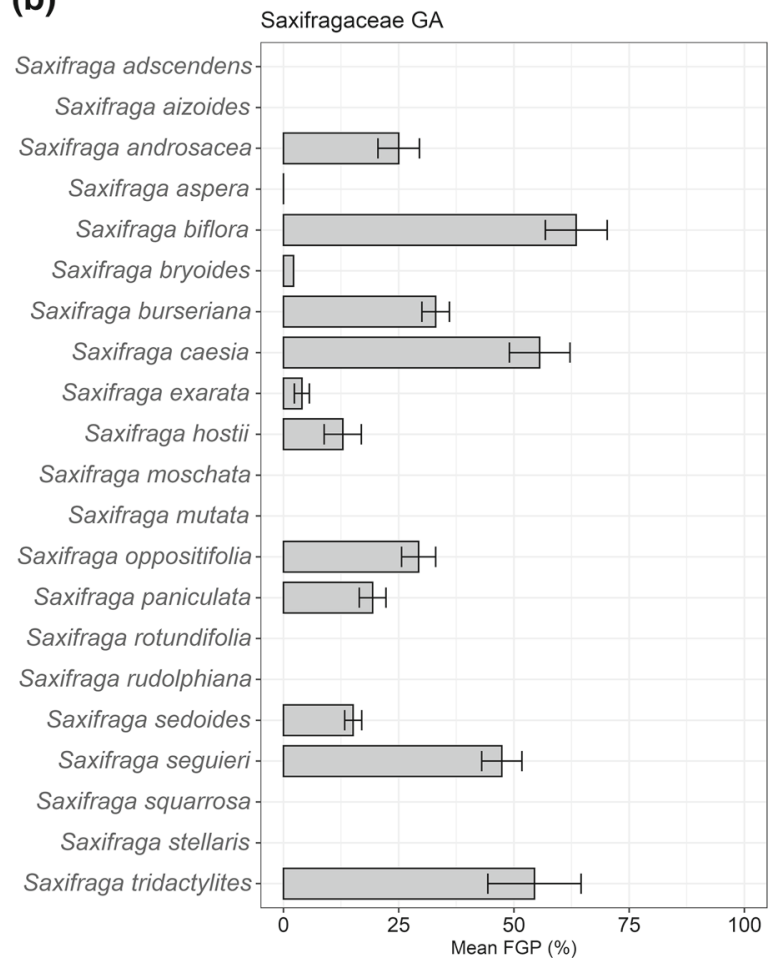

Fig. 6 a Final germination percentage (FGP) of 21 species from the family Saxifragaceae, tested under the standard protocol and $\mathbf{b}$ FGP after a treatment with gibberellic acid (GA). These species were cold-wet stratified before the germination test

et al. 2009). In particular, Ranunculaceae have rudimentary underdeveloped embryos (FinchSavage and Leubner-Metzger 2006). Noccaea crantzii and Pedicularis portenschlagii, both endemic species, were stimulated by $\mathrm{GA}_{3}$, however, for other endemic species (i.e. Valeriana species) we did not have enough seeds to perform the $\mathrm{GA}_{3}$ treatment and it remains unknown if the dormancy of these species could have been broken by a $\mathrm{GA}_{3}$ treatment. Further, some endemic species require a cold-wet stratification (i.e. 90 days for an endemic Telekia, Brusa et al. 2007; at least nine months for an endemic Androsace, Frattaroli et al. 2013), whereas for an endemic Campanula, $24 \mathrm{~h}$ seed imbibition and a specific germination medium is required (Frattaroli et al. 2013). These examples and our results illustrate that no generalisations for $\mathrm{GA}_{3}$ treatment are possible, due to the individual germination response of endemic species. Moreover, even between individuals of the same population, dormancy may vary
(Andersson and Milberg 1998). The loss of physiological dormancy may be closely related to the environment of the maternal habitat (Rosbakh and Poschlod 2015). Thus, it would be advisable to test a variety of temperature regimes for germination in combination with seed pre-treatments.

(h) Among the studied species with weak or zero germination, only few might have had physical dormancy. This mechanism is typical for Fabaceae and for sure, scarification might have been an efficient dormancy release for species from this family (Schütz 1988; Flüeler 2011; Schwienbacher et al. 2011). However, we did not consider such treatments in our screening project. Asteraceae and Poaceae are among the most widespread families in the Alps (Körner 2003). According to our results, most species of these two families germinated to a high extent using the standard protocol, i.e. at warm temperatures $\left(20 / 10{ }^{\circ} \mathrm{C}\right.$ or $\left.25 / 15^{\circ} \mathrm{C}\right)$. This means, higher temperatures in the field that occur due to 
climate warming may be tolerated for germination without problems, which may lead to increases in seedling numbers, and may turn into increased seedling recruitment. This hypothesis is in line with several statements made already for Poaceae (Venn et al. 2014; Porro et al. 2019). In contrast, species from the family Saxifragaceae seemed to have problems with the conditions of the standard protocol, although Schwienbacher et al. (2011) recorded an optimum germination temperature of $20 / 10{ }^{\circ} \mathrm{C}$ for alpine Saxifraga species. Their germination might be restricted by different types of dormancy. However, after a stratification period of three month, some species did still not germinate, and several were stimulated only weakly by a $\mathrm{GA}_{3}$ treatment. Giménez-Benavides and Milla (2013) even noted a negative effect of cold-wet stratification on germination success on two Saxifraga species from northern Spain. The only annual species among the collected, $S$. tridactylites, did not germinate under the standard protocol. It needs low temperatures of $5{ }^{\circ} \mathrm{C}$ and $10{ }^{\circ} \mathrm{C}$ for germination (Pemadasa and Lovell 1975) while high temperatures enforce dormancy. This is a nice example of germination temperature reflecting adaptation to habitat conditions of winter annuals.

Importance of findings for ex situ seed conservation and seedling recruitment in the field

In any classification in this study, about half of the species germinated up to intermediate and high extents using the standard protocol. All the species tested originated from the European Eastern Alps. It seems that most Alpine species require relatively high temperatures for germination, but that germination is restricted by different types of dormancy. For ex situ seed banking, an application of different dormancy release treatments is advisable when testing germination of Alpine species. However, for rare and endemic species, seeds for several parallel trials are often limited or not available. In these cases, standard protocols must be applied, and for Alpine species, a high temperature regime might be recommended.
Seedling recruitment is a highly risky developmental stage in nival-alpine environments (Stöcklin and Bäumler 1996; Erschbamer et al. 2008; Marcante et al. 2009; Erschbamer and Caccianiga 2017). Therefore, high germination rates are mandatory for alpine plants to persist or migrate. The same holds for upwardmigrating species along elevation gradients. Climate warming may enhance germination as shown for arctic species (Alsos et al. 2013). However, according to niche models (Schwager and Berg 2019) habitat suitability will change remarkably, and several Alpine species might be suffering from habitat loss. Germination experiments in the laboratory are valuable, however, they do not substitute for observations in the field under real environmental conditions. Investigations of the reproductive output, seedling survival and recruitment, as well as the demographic behaviour of species should be focussed by future projects.

Acknowledgements Open access funding provided by University of Innsbruck and Medical University of Innsbruck. We are thankful to all seed collectors (Monika Hamacher, Andreas Bär, Bettina Mittendrein, Cäcilia Lechner-Pagitz, Lena Nicklas, Moritz Falch, Martina Pöltl, Matthias Kaltenböck), helping hands in the lab (Barbara Pernfuß, Charlotte Permann, Katharina Ramskogler) and to Matthias Hepp for the germination tests of the seed collection 2017. Further, we are grateful to two anonymous reviewers for their valuable comments on the manuscript.

Funding This study was done as part of the "Alpine Seed Conservation and Research Network" (https://www. alpineseedconservation.eu/), a project managed by the Royal Botanic Gardens, Kew, funded by the David and Claudia Harding Foundation. VM was additionally supported by a $\mathrm{PhD}$ scholarship grant from the Vice rector for research at the University of Innsbruck.

Data availability The data are currently not on a repository. The datasets are available from the corresponding author on reasonable request.

\section{Compliance with ethical standards}

Conflict of interest The authors declare that they have no conflicts of interest.

Ethical approval The study did not cause any harm to the environment, animals, or plants. The study did not involve human participants and/or animals.

Informed Consent The publication in Plant Ecology has been approved by all co-authors. 
Open Access This article is licensed under a Creative Commons Attribution 4.0 International License, which permits use, sharing, adaptation, distribution and reproduction in any medium or format, as long as you give appropriate credit to the original author(s) and the source, provide a link to the Creative Commons licence, and indicate if changes were made. The images or other third party material in this article are included in the article's Creative Commons licence, unless indicated otherwise in a credit line to the material. If material is not included in the article's Creative Commons licence and your intended use is not permitted by statutory regulation or exceeds the permitted use, you will need to obtain permission directly from the copyright holder. To view a copy of this licence, visit http://creativecommons.org/licenses/by/4.0/.

\section{References}

Alexander JM, Diez JM, Levine JM (2015) Novel competitors shape species' responses to climate change. Nature 525:515-518. https://doi.org/10.1038/nature14952

Alexander JM, Chalmandrier L, Lenoir J, Burgess TI, Essl F, Haider S, Kueffer C, McDougall K, Milbau A, Nuñez MA, Pauchard A, Rabitsch W, Rew LJ, Sanders NJ, Pellissier L (2018) Lags in the response of mountain plant communities to climate change. Glob Chang Biol 24:563-579. https:// doi.org/10.1111/gcb.13976

Alsos IG, Müller E, Eidesen PB (2013) Germinating seeds or bulbils in 87 of 113 tested arctic species indicate potential for ex situ seed bank storage. Polar Biol 36:819-830. https://doi.org/10.1007/s00300-013-1307-7

Amen RD (1966) The extent and role of seed dormancy in alpine plants. Q Rev Biol 41:271-281. https://doi.org/10.1086/ 405055

Andersson L, Milberg P (1998) Variation in seed dormancy among mother plants, populations and years of seed collection. Seed Sci Res 8:29-38. https://doi.org/10.1017/ s0960258500003883

Auguie B, Antonov A (2017) Package gridExtra. https://cran.rproject.org/web/packages/gridExtra/gridExtra.pdf. Accessed 14 Nov 2019

Baskin JM, Baskin CC (2004) A classification system for seed dormancy. Seed Sci Res 14:1-16. https://doi.org/10.1079/ ssr2003150

Baskin CC, Baskin JM (2005) Underdeveloped embryos in dwarf seeds and implications for assignment to dormancy class. Seed Sci Res 15:357-360. https://doi.org/10.1079/ ssr2005224

Baskin CC, Baskin JM (2014) Seeds: ecology, biogeography, and evolution of dormancy and germination, 2nd edn. Elsevier, London

Bernareggi G, Carbognani M, Mondoni A, Petraglia A (2016) Seed dormancy and germination changes of snowbed species under climate warming: the role of pre-and postdispersal temperatures. Ann Bot 118:529-539. https://doi. org/10.1093/aob/mcw125
Billings WD, Mooney HA (1968) The ecology of arctic and alpine plants. Biol Rev 43:481-529. https://doi.org/10. 1111/j.1469-185X.1968.tb00968.x

Bliss LC (1958) Seed germination in arctic and alpine species. Arctic 11:180. https://doi.org/10.14430/arctic3743

Brusa G, Ceriani R, Cerabolini B (2007) Seed germination in a narrow endemic species (Telekia speciosissima, Asteraceae): implications for ex situ conservation. Plant Biosyst 141:56-61. https://doi.org/10.1080/11263500601153495

Bu H, Chen X, Xu X, Liu K, Jia P, Du G (2007) Seed mass and germination in an alpine meadow on the eastern TsinghaiTibet plateau. Plant Ecol 191:127-149. https://doi.org/10. 1007/s11258-006-9221-5

Caccianiga M, Luzzaro A, Pierce S, Ceriani RM, Cerabolini B (2006) The functional basis of a primary succession resolved by CSR classification. Oikos 112:10-20. https:// doi.org/10.1111/j.0030-1299.2006.14107.x

Casazza G, Giordani P, Benesperi R, Foggi B, Viciani D, Filigheddu R, Farris E, Bagella S, Pisanu S, Mariotti MG (2014) Climate change hastens the urgency of conservation for range-restricted plant species in the central-northern Mediterranean region. Biol Conserv 179:129-138. https:// doi.org/10.1016/j.biocon.2014.09.015

Cavieres LA, Arroyo MTK (2000) Seed germination response to cold stratification period and thermal regime in Phacelia secunda (Hydrophyllaceae): altitudinal variation in the mediterranean andes of central Chile. Plant Ecol 149:1-8. https://doi.org/10.1023/A:1009802806674

Cavieres LA, Sierra-Almeida A (2018) Assessing the importance of cold-stratification for seed germination in alpine plant species of the High-Andes of central Chile. Perspect Plant Ecol Evol Syst 30:125-131. https://doi.org/10.1016/ j.ppees.2017.09.005

Coomes DA, Grubb PJ (2003) Colonization, tolerance, competition and seed-size variation within functional groups. Trends Ecol Evol 18:283-291. https://doi.org/10.1016/ S0169-5347(03)00072-7

Donohue K, Rubio de Casas R, Burghardt L, Kovach K, Willis CG (2010) Germination, postgermination adaptation, and species ecological ranges. Annu Rev Ecol Evol Syst 41:293-319. https://doi.org/10.1146/annurev-ecolsys102209-144715

ENSCONET (2009a) ensconet seed collecting manual for wild species. https://www.publicgardens.org/resources/ ensconet-seed-collecting-manual-wild-species. Accessed 4 Feb 2020

ENSCONET (2009b) ENSCONET Curation protocols \& Recommendations. https://ensconet.maich.gr/PDF/Curation_ protocol_English.pdf. Accessed 4 Feb 2020

Eriksson O (1999) Seed size variation and its effect on germination and seedling performance in the clonal herb Convallaria majalis. Acta Oecol 20:61-66. https://doi.org/10. 1016/S1146-609X(99)80016-2

Erschbamer B, Pfattner M (2002) Das Keimverhalten von alpinen Arten in der Klimakammer und im Gelände Germination characteristics of alpine species in the growth chamber and in the field site. Ber Nat Med Verein Innsbruck 89:87-97

Erschbamer B, Caccianiga MS (2017) Glacier forelands: lessons of plant population and community development. In: 
Cánovas FM, Lüttge U, Matyssek R (eds) Progress in botany, vol 78. Springer, Cham, pp 259-284

Erschbamer B, Niederfriniger Schlag R, Winkler E (2008) Colonization processes on a central alpine glacier foreland. J Veg Sci 19:855-862. https://doi.org/10.3170/2008-818464

Erschbamer B, Anich C, Benirschke M, Ganthaler A, Grassmair R, Hasibeder R, Huter V, Konzett D, Lechleitner M, Magauer M, Miller R, Newerkla S, Schneider J, Zeisler B, Schwienbacher E (2010) Das Keimverhalten von 13 alpinen Arten der Familie Asteraceae im Licht und im Dunkeln. Ber Nat-Med Verein Innsbruck 96:73-78

Fenner M, Thompson K (2005) The ecology of seeds, 1st edn. Cambridge University Press, Cambridge

Fernández-Pascual E, Jiménez-Alfaro B, Bueno A (2017) Comparative seed germination traits in alpine and subalpine grasslands: higher elevations are associated with warmer germination temperatures. Plant Biol 19:32-40. https://doi.org/10.1111/plb.12472

Finch-Savage WE, Leubner-Metzger G (2006) Seed dormancy and the control of germination. New Phytol 171:501-523. https://doi.org/10.1111/j.1469-8137.2006.01787.x

Fischer MA, Oswald K, Adler W (2008) Exkursionsflora von Österreich, Liechtenstein und Südtirol. Biologiezentrum der Oberösterreichischen Landesmuseen, 3rd edn. Land Oberösterreich, Linz

Flüeler RP (2011) Experimentelle Untersuchungen über Keimung und Etablierung von alpinen Leguminosen. Diss Veröff Geobt Inst ETH Stift Rübel Zürich. https://doi.org/ 10.3929/ethz-a-000643314

Forbis TA (2003) Seedling demography in an Alpine ecosystem. Am J Bot 90:1197-1206. https://doi.org/10.3732/ajb.90.8. 1197

Forbis TA, Diggle PK (2001) Subnivean embryo development in the alpine herb Caltha leptosepala (Ranunculaceae). Can J Bot 79:635-642. https://doi.org/10.1139/b01-037

Frattaroli AR, Di Martino L, Di Cecco V, Catoni R, Varone L, Di Santo M, Gratani L (2013) Seed germination capability of four endemic species in the Central Apennines (Italy): relationships with seed size. Lazaroa 34:43-53. https://doi. org/10.5209/rev_LAZA.2013.v34.n1.42253

García-Fernández A, Escudero A, Lara-Romero C, Iriondo JM (2015) Effects of the duration of cold stratification on early life stages of the Mediterranean alpine plant Silene ciliata. Plant Biol 17:344-350. https://doi.org/10.1111/plb.12226

Giménez-Benavides L, Milla R (2013) Comparative germination ecology of two altitudinal vicariant Saxifraga species endemic to the north of Spain. Plant Biol 15:593-600. https://doi.org/10.1111/j.1438-8677.2012.00663.x

Giménez-Benavides L, Escudero A, Pérez-García F (2005) Seed germination of high mountain Mediterranean species: altitudinal, interpopulation and interannual variability. Ecol Res 20:433-444. https://doi.org/10.1007/s11284005-0059-4

Gobiet A, Kotlarski S, Beniston M, Heinrich G, Rajczak J, Stoffel M (2014) 21st century climate change in the European Alps-a review. Sci Total Environ 493:1138-1151. https://doi.org/10.1016/j.scitotenv.2013. 07.050

Graae BJ, Alsos IG, Ejrnaes R (2008) The impact of temperature regimes on development, dormancy breaking and germination of dwarf shrub seeds from arctic, alpine and boreal sites. Plant Ecol 198:275-284. https://doi.org/10. 1007/s11258-008-9403-4

Graae BJ, Ejrnæs R, Lang SI, Meineri E, Ibarra PT, Bruun HH (2011) Strong microsite control of seedling recruitment in tundra. Oecologia 166:565-576. https://doi.org/10.1007/ s00442-010-1878-8

Grime JP (1979) Plant strategies and vegetation processes. Wiley, Chichester

Grosjean P, Ibanez Frederic, Etienne M (2018) Package 'pastecs': package for analysis of space-time ecological series. https://cran.r-project.org/web/packages/pastecs/pastecs. pdf. Accessed 14 Nov 2019

Grubb PJ (1977) The maintenance of species-richness in plant communities: the importance of the regeneration niche. Biol Rev 52:107-145. https://doi.org/10.1111/j.1469185x.1977.tb01347.x

Harper JL (1977) Population biology of plants. Academic Press, London

Isselin-Nondedeu F, Bédécarrats A (2013) Germination and seedling responses of subalpine plants to different soil substrates. Folia Geobot 48:39-53. https://doi.org/10. 1007/s12224-012-9136-y

Jiménez-Alfaro B, Silveira FAO, Fidelis A, Poschlod P, Commander LE (2016) Seed germination traits can contribute better to plant community ecology. J Veg Sci 27:637-645. https://doi.org/10.1111/jvs.12375

Kawecki TJ, Ebert D (2004) Conceptual issues in local adaptation. Ecol Lett 7:1225-1241. https://doi.org/10.1111/j. 1461-0248.2004.00684.x

Körner C (2003) Alpine plant life. Functional plant ecology of high mountain ecosystems. Springer, Berlin

Landolt E, Bäumler B, Erhardt A, Hegg O, Klötzli F, Lämmler W, Nobis M, Rudmann-Maurer K, Schweingruber FH, Theurillat J-P, Urmi E, Vust M, Wohlgemuth T (2010) Flora indicativa. Ecological indicator values and biological attributes of the Flora of Switzerland and the Alps, 2nd edn. Haupt, Bern

Liu K, Baskin JM, Baskin CC, Bu H, Du G, Ma M (2013) Effect of diurnal fluctuating versus constant temperatures on germination of 445 species from the Eastern Tibet Plateau. PLoS ONE. https://doi.org/10.1371/journal.pone.0069364

Marcante S, Winkler E, Erschbamer B (2009) Population dynamics along a primary succession gradient: do alpine species fit into demographic succession theory? Ann Bot 103:1129-1143. https://doi.org/10.1093/aob/mcp047

Margreiter V, Walde J, Erschbamer B (in review) Germination and seedling recruitment of alpine species in a two-year field experiment along an elevation gradient

Milbau A, Graae BJ, Shevtsova A, Nijs I (2009) Effects of a warmer climate on seed germination in the subarctic. Ann Bot 104:287-296. https://doi.org/10.1093/aob/mcp117

Milbau A, Vandeplas N, Kockelbergh F, Nijs I (2017) Both seed germination and seedling mortality increase with experimental warming and fertilization in a subarctic tundra. AoB Plants 9:1-13. https://doi.org/10.1093/aobpla/plx040

Moles AT, Westoby M (2006) Seed size and plant strategy across the whole life cycle. Oikos 113:91-105. https://doi. org/10.1111/j.0030-1299.2006.14194.x

Mondoni A, Daws MI, Belotti J, Rossi G (2009) Germination requirements of the alpine endemic Silene elisabethae Jan: 
effects of cold stratification, light and GA3. Seed Sci Technol 37:79-87. https://doi.org/10.15258/sst.2009.37.1. 10

Mondoni A, Probert RJ, Rossi G, Vegini E, Hay FR (2011) Seeds of alpine plants are short lived: Implications for long-term conservation. Ann Bot 107:171-179. https://doi. org/10.1093/aob/mcq222

Mondoni A, Orsenigo S, Müller JV, Carlsson-Graner U, Jiménez-Alfaro B, Abeli T (2018) Seed dormancy and longevity in subarctic and alpine populations of Silene suecica. Alp Bot 128:71-81. https://doi.org/10.1007/s00035-017-0194$\mathrm{x}$

Mondoni A, Rossi G, Orsenigo S, Probert RJ (2012) Climate warming could shift the timing of seed germination in alpine plants. Ann Bot 110:155-164. https://doi.org/10. 1093/aob/mcs097

Müller JV, Berg C, Détraz-Méroz J, Erschbamer B, Fort N, Lambelet-Haueter C, Margreiter V, Mombrial F, Mondoni A, Pagitz K, Porro F, Rossi G, Schwager P, Breman E (2017) The alpine seed conservation and research network-a new initiative to conserve valuable plant species in the European Alps. J Mt Sci 14:806-810. https://doi.org/ 10.1007/s11629-016-4313-8

Orsenigo S, Abeli T, Rossi G, Bonasoni P, Pasquaretta C, Gandini M, Mondoni A (2015) Effects of autumn and spring heat waves on seed germination of high mountain plants. PLoS ONE. https://doi.org/10.1371/journal.pone. 0133626

Pemadasa MA, Lovell PH (1975) Factors controlling germination of some dune annuals. J Ecol 63:41-59

Pierce S, Bottinelli A, Bassani I, Ceriani RM, Cerabolini BEL (2014) How well do seed production traits correlate with leaf traits, whole-plant traits and plant ecological strategies? Plant Ecol 215:1351-1359. https://doi.org/10.1007/ s11258-014-0392-1

Porro F, Tomaselli M, Abeli T, Gandini M, Gualmini M, Orsenigo S, Petraglia A, Rossi G, Carbognani M (2019) Could plant diversity metrics explain climate-driven vegetation changes on mountain summits of the GLORIA network? Biodivers Conserv 28:3575-3596. https://doi. org/10.1007/s10531-019-01837-1

$\mathrm{R}$ Core Team (2020) A language and environment for statistical computing. In: R Found. Stat. Comput. https://www.rproject.org. Accessed 15 May 2020

Rosbakh S, Poschlod P (2015) Initial temperature of seed germination as related to species occurrence along a temperature gradient. Funct Ecol 29:5-14. https://doi.org/10. $1111 / 1365-2435.12304$

RStudio RsT (2016) Integrated development for R. RStudio. In: RStudio, Inc., Boston, MA. https://www.rstudio.com/. Accessed 15 May 2020

Schütz M (1988) Genetisch-ökologische Untersuchungen an alpinen Pflanzenarten auf verschiedenen Gesteinsunterlagen : Keimungs- und Aussaatversuche $=$ Genetic-ecological studies in alpine plants from various substrata : germination trials and sowing experiments. Veröffentlichungen des Geobot Institutes der Eidg Tech Hochschule, Stift Rübel Zürich, pp 963027-963032

Schütz W, Milberg P, Schutz W (1997) Seed dormancy in carex canescens: regional differences and ecological consequences. Oikos 78:420. https://doi.org/10.2307/3545604
Schwager P, Berg C (2019) Global warming threatens conservation status of alpine EU habitat types in the European Eastern Alps. Reg Environ Chang 19:2411-2421. https:// doi.org/10.1007/s10113-019-01554-z

Schwienbacher E, Erschbamer B (2001) Longevity of seeds in a glacier foreland of the Central Alps-a burial experiment. Bull Geobot Inst ETH 68:63-71. https://doi.org/10.5169/ seals-377848

Schwienbacher E, Navarro-Cano JA, Neuner G, Erschbamer B (2011) Seed dormancy in alpine species. Flora Morphol Distrib Funct Ecol Plants 206:845-856. https://doi.org/10. 1016/j.flora.2011.05.001

Schwienbacher E, Navarro-Cano JA, Neuner G, Erschbamer B (2012) Correspondence of seed traits with niche position in glacier foreland succession. Plant Ecol 213:371-382. https://doi.org/10.1007/s11258-011-9981-4

Shevtsova A, Graae BJ, Jochum T, Milbau A, Kockelbergh F, Beyens L, Nijs I (2009) Critical periods for impact of climate warming on early seedling establishment in subarctic tundra. Glob Chang Biol 15:2662-2680. https://doi.org/10. $1111 /$ j.1365-2486.2009.01947.x

Shimono Y, Kudo G (2003) Intraspecific variations in seedling emergence and survival of Potentilla matsumurae (Rosaceae) between alpine fellfield and snowbed habitats. Ann Bot 91:21-29. https://doi.org/10.1093/aob/mcg002

Stöcklin J, Bäumler E (1996) Seed rain, seedling establishment and clonal growth strategies on a glacier foreland. J Veg Sci 7:45-56. https://doi.org/10.2307/3236415

Thompson K, Gastonn KJ, Band SR (1999) Range size, dispersal and niche breadth in the herbaceous flora of central England. J Ecol 87:150-155. https://doi.org/10.1046/j. 1365-2745.1999.00334.x

Threadgill PF, Baskin JM, Baskin CC (1981) Dormancy in seeds of Frasera caroliniensis (Gentianaceae). Am J Bot 68:80-86. https://doi.org/10.1002/j.1537-2197.1981. tb06358.x

Tilman D (1994) Competition and biodiversity in spatially structured habitats. Ecology 75:2-16. https://doi.org/10. 2307/1939377

Tudela-Isanta M, Fernández-Pascual E, Wijayasinghe M, Orsenigo S, Rossi G, Pritchard HW, Mondoni A (2018) Habitat-related seed germination traits in alpine habitats. Ecol Evol 8:150-161. https://doi.org/10.1002/ece3.3539

Tudela-Isanta M, Ladouceur E, Wijayasinghe M, Pritchard HW, Mondoni A (2018) The seed germination niche limits the distribution of some plant species in calcareous or siliceous alpine bedrocks. Alp Bot 128:83-95. https://doi.org/10. 1007/s00035-018-0199-0

Vandelook F, Bolle N, Van Assche JA (2009) Morphological and physiological dormancy in seeds of aegopodium podagraria (apiaceae) broken successively during cold stratification. Seed Sci Res 19:115-123. https://doi.org/10. 1017/S0960258509301075

Venables WN, Ripley BD (2002) Modern applied statistics with $\mathrm{S}, 4$ th edn. Springer, Berlin

Venn S, Pickering C, Green K (2014) Spatial and temporal functional changes in alpine summit vegetation are driven by increases in shrubs and graminoids. AoB Plants. https:// doi.org/10.1093/aobpla/plu008

Vera ML (1997) Effects of altitude and seed size on germination and seedling survival of heathland plants in north spain. 
Plant Ecol 133:101-106. https://doi.org/10.1023/A: 1009729201384

Walder T, Erschbamer B (2015) Temperature and drought drive differences in germination responses between congeneric species along altitudinal gradients. Plant Ecol 216:1297-1309. https://doi.org/10.1007/s11258-0150509-1

Wang JH, Baskin CC, Chen W, Du GZ (2010) Variation in seed germination between populations of five sub-alpine woody species from eastern Qinghai-Tibet Plateau following dry storage at low temperatures. Ecol Res 25:195-203. https:// doi.org/10.1007/s11284-009-0643-0

Wenk EH, Dawson TE (2007) Interspecific differences in seed germination, establishment, and early growth in relation to preferred soil type in an alpine community. Arctic Antarct Alp Res 39:165-176. https://doi.org/10.1657/15230430(2007)39[165:IDISGE]2.0.CO;2

Westoby M (1998) A leaf-height-seed (LHS) plant ecology strategy scheme. Plant Soil 199:213-227. https://doi.org/ 10.1023/A: 1004327224729

Wickham H (2017) Package 'tidyverse'. In: CRAN repos. https://tidyverse.org. Accessed 15 May 2020

Wickham H (2018a) Package 'reshape' flexibly reshape data. https://had.co.nz/reshape/. Accessed 15 May 2020
Wickham H (2018b) Package 'scales'-scale functions for visualization. In: CRAN Repos. https://cran.r-project.org/ web/packages/scales/scales.pdf. Accessed 15 May 2020

Wickham H, Winston C (2019) create elegant data visualisations using the grammar of graphics. In: Packag. 'ggplot2'. https://cran.r-project.org/web/packages/ggplot2/ggplot2. pdf. Accessed 15 May 2020

Winkler E, Marcante S, Erschbamer B (2015) Demography of the alpine pioneer species Saxifraga aizoides in different successional stages at the glacier fore-land of the Rotmoosferner (Obergurgl, ötztal, Austria). Tuexenia 35:267-283. https://doi.org/10.14471/2015.35.002

ZAMG (2016) Jahresbericht/Annual report. https://www.zamg. ac.at/cms/de/topmenu/ueber-uns/jahresberichte. Accessed 29 June 2020

ZAMG (2017) Jahresbericht/Annual report. https://www.zamg. ac.at/cms/de/topmenu/ueber-uns/jahresberichte. Accessed 29 June 2020

ZAMG (2018) Jahresbericht/Annual report. https://www.zamg. ac.at/cms/de/topmenu/ueber-uns/jahresberichte. Accessed 29 June 2020

Publisher's Note Springer Nature remains neutral with regard to jurisdictional claims in published maps and institutional affiliations. 